\title{
Contribution of solitons to enhanced rogue wave occurrence in shallow water: a case study in the southern North Sea
}

\author{
Ina Teutsch ${ }^{1}$, Markus Brühl ${ }^{2}$, Ralf Weisse ${ }^{1}$, and Sander Wahls ${ }^{2}$ \\ ${ }^{1}$ Helmholtz-Zentrum Hereon, Max-Planck-Str. 1, 21502 Geesthacht, Germany \\ ${ }^{2}$ Delft Center for Systems and Control, Delft University of Technology, 2860 Delft, South Holland, Netherlands
}

Correspondence: Ina Teutsch (ina.teutsch@hereon.de)

\begin{abstract}
The shallow waters off the coast of Norderney in the southern North Sea are characterised by a higher frequency of rogue wave occurrences than expected according to second-order theory. The role of nonlinear processes for the generation of rogue waves at this location is currently unclear. Within the framework of the Korteweg-de Vries (KdV) equation, we investigated the discrete soliton spectra of measured time series at Norderney to determine differences between time series with and without rogue waves. For this purpose, we applied a nonlinear Fourier transform for the Korteweg-de Vries equation with vanishing boundary conditions (vKdV-NLFT). For each time series containing a rogue wave, we were able to identify at least one soliton in the discrete nonlinear vKdV-NLFT spectrum that contributed to the occurrence of the rogue wave in that time series. The amplitudes of these solitons were generally found to be smaller than the crest height of the corresponding rogue wave and interaction with the continuous wave spectrum is needed to fully explain the observed rogue wave. Time series with and without rogue waves showed different characteristic soliton spectra. In most of the spectra calculated from rogue wave time series, most of the solitons clustered around similar heights, while the largest soliton was outstanding with an amplitude significantly larger than all other solitons. The presence of a clearly outstanding soliton in the spectrum was found to be an indicator pointing towards enhanced probability for detecting a rogue wave in the time series. Similarly, when the discrete spectrum appears as a cluster of solitons without the presence of a clearly outstanding soliton, the presence of a rogue wave in the observed time series is unlikely. Under the hypothesis that the KdV describes the evolution of the sea state around the measurement site well, these results suggest that solitons and nonlinear processes substantially contribute to the enhanced occurrence of rogue waves off Norderney.
\end{abstract}

\section{Introduction}

There has been a lively discussion on whether the occurrence frequency of rogue waves in the open ocean is well described by second-order models. Both Rayleigh (Longuet-Higgins, 1952) and Weibull distributions (Forristall, 1978) have been used to describe the distributions of wave and crest heights. Distributions were assessed for measurement data collected by surfacefollowing buoys (e.g., Baschek and Imai, 2011; Pinho et al., 2004; Cattrell et al., 2018), by radar devices (e.g., Olagnon and v. Iseghem, 2000; Christou and Ewans, 2014; Karmpadakis et al., 2020), and laser altimeters (e.g., Soares et al., 2003; Stansell, 2004), as well as by ADCPs (Fedele et al., 2019). Independent of the measurement device, some authors found measured 
https://doi.org/10.5194/nhess-2022-28

Preprint. Discussion started: 4 March 2022

(C) Author(s) 2022. CC BY 4.0 License.

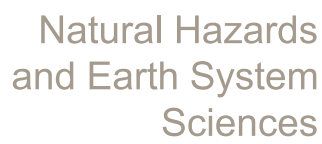

Discussions

25 wave heights to agree well with the established distributions, while others found the frequency of rogue wave occurrences over- or underestimated. For example, for the northern North Sea, all three inferences have been made: While Olagnon and v. Iseghem (2000) found rogue wave occurrences to be overpredicted by the classical distributions, they appeared to be in agreement with a Weibull distribution in data of Waseda et al. (2011) and to be underestimated in the dataset of Stansell (2004). Rogue wave occurrences in buoy data from the US coast, recorded in shallow, intermediate and deep water, were found to be strongly overestimated by a Rayleigh distribution. Furthermore, the respective authors describe local differences in rogue wave occurrence frequency between their measurement stations (Baschek and Imai, 2011), depending on the wave climate and especially in coastal waters, where waves interact with the seabed (Cattrell et al., 2018; Orzech and Wang, 2020). In a previous study, we have analysed measurement data from various stations in the southern North Sea (Teutsch et al., 2020) and found rogue wave frequencies to vary spatially and by measurement device. For data obtained from wave buoy measurements, we generally found rogue wave frequencies slightly overestimated by the Forristall distribution, which is a special form of the Weibull distribution, fit to wave data recorded during hurricanes (Forristall, 1978). An exception was one measurement buoy, which was located in the shallow waters off the coast of the island Norderney, Germany (Fig. 1). For this buoy, enhanced rogue wave occurrence, which could not be explained by the Forristall distribution, was observed. This suggests that nonlinear processes and interactions may play a role in explaining the increase in rogue wave occurrence frequency at this specific location. 


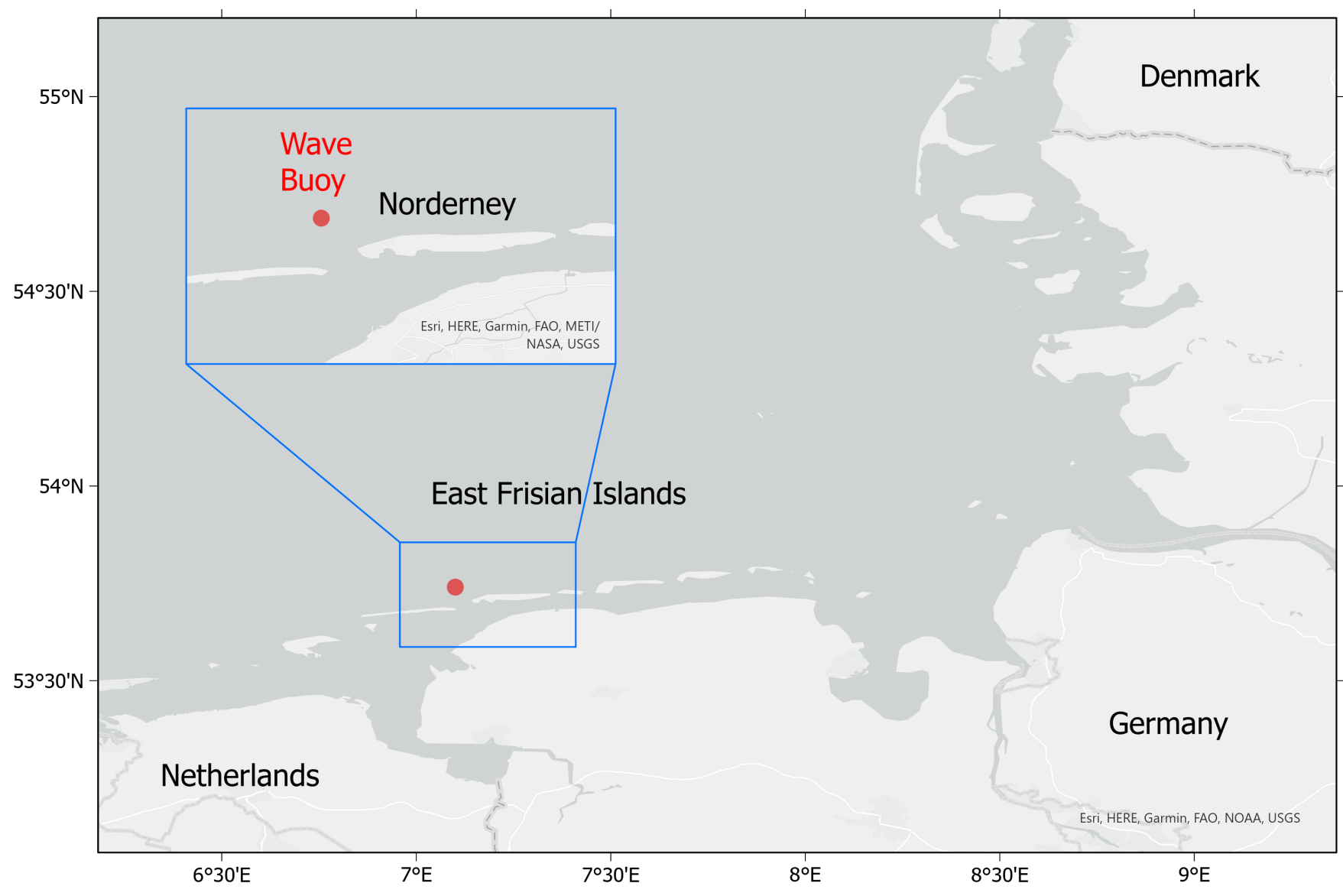

Figure 1. Map of the German Bight, showing the location of the measurement buoy close to the island Norderney.

So far, the nonlinear behaviour of deep-water rogue waves has received considerably more attention than that of shallowwater rogue waves. The evolution of the complex envelope of unidirectional wave trains in deep water can be described by the cubic nonlinear Schroedinger (NLS) equation (Onorato et al., 2001; Slunyaev, 2005). Deep water in this context is defined in terms of the wave number $k$ and the water depth $h$ as $k h>1.36$, which represents the lower limit for the application of the NLS equation (Osborne, 2010). The NLS equation is a weakly nonlinear, narrow-banded approximation of the fully nonlinear water wave equations, including both nonlinearity and dispersion (Serio et al., 2006). In deep water, rogue-wave occurrence beyond the second-order model has been explained, for example, by a nonlinear instability that was also found in numerical simulations and tank experiments (Dysthe et al., 2008). Here, uniform wave trains are modulationally unstable to small oblique perturbations and disintegrate into groups, in which the highest wave becomes significantly larger than the wave height in the original train (Benjamin and Feir, 1967). This nonlinear focusing mechanism does not only increase the maximum wave height, but also the probability of rogue wave occurrence (Slunyaev and Shrira, 2013). Alber (1978) derived a stability criterion for such narrow-banded random waves, which later became known as the Benjamin-Feir index (BFI) (Janssen, 2003). A large BFI corresponds to enhanced nonlinearity (de León and Soares, 2014) and has been suggested as an indicator for enhanced rogue 
wave probability in deep water (Gramstad and Trulsen, 2007). The NLS equation has some exact solutions (known as breathers) that explain the modulational instability, which have been suggested as an analytical model of rogue waves in a unidirectional case (Dysthe and Trulsen, 1999). Here a uniform wave train develops into a number of breathers, and then relaxes back to a uniform wavetrain (Clamond et al., 2006; Gramstad and Trulsen, 2007). Each breather solution represents the modulational instability growth for a specific initial perturbation. In the framework of the NLS equation, a large part of the dynamics of nonlinear waves can be described in terms of interacting breathers (Slunyaev and Shrira, 2013). Specifically the Peregrine breather (Peregrine, 1983), which is characterised by only one oscillation in time and an amplitude of three times the initial wave train, has been subject to analysis (Shrira and Geogjaev, 2009). Recently, the growth of crest heights due to nonlinearities that was observed in deep water, has been extended to intermediate water depths (Karmpadakis et al., 2019). However, the relevance of the modulational instability of the NLS equation to the formation of real rogue waves remains unclear because most of these works only consider the specific scenario of perturbed plane wave envelopes (Slunyaev and Shrira, 2013). The role of nonlinearity with respect to rogue wave generation in shallow water has received considerably less attention than for deep water. Shallow-water wind waves substantially differ from deep-water wind waves and it is not appropriate to simply scale the deep-water nonlinear interaction to shallow-water waves (Janssen and Onorato, 2007). As the water depth becomes more and more shallow, a wave-induced current develops and less wave energy is available for nonlinear focusing (Benjamin and Feir, 1967; Janssen and Onorato, 2007). Although waves in shallow water can also destabilise due to oblique perturbations (Toffoli et al., 2013), the modulational instability in shallow water does not enhance the formation of extreme waves (Fernandez et al., 2014). Fedele et al. (2019) stated that waves in shallow water break before they can start to "breathe" and become rogue waves. Therefore, some authors expect the rogue wave probability to decrease in shallow water (e.g., Slunyaev et al., 2016). Other authors refer to the large ratio between nonlinearity and dispersion in shallow water (Kharif and Pelinovsky, 2003) and concluded that Gaussian statistics are not sufficient for the description of shallow-water waves and that rogue waves are likely to occur more frequently as the water depth decreases (Garett and Gemmrich, 2009; Sergeeva et al., 2011). While in deep water only the free-surface nonlinearity must be taken into account, the nonlinearity in shallow water is mainly a result of the interaction of waves with the sea floor (Prevosto, 1998). Refraction, shoaling and higher-order nonlinear effects change the shapes of waves and their energy spectrum (Bitner, 1980; Tayfun, 2008). However, so far only few studies have addressed the impact of bathymetry on rogue wave generation. For example, Soomere (2010) found that in shallow water, compared to deep water, due to wave-bathymetry interaction, additional processes associated with the generation of extreme waves, like wave amplification along certain coastal profiles, redirection of waves or the formation of crossing seas, are relevant, and therefore more rogue waves should be expected in nearshore regions.

The shallow-water equivalent to the NLS equation is the Korteweg-de Vries (KdV) equation (Korteweg and de Vries, 1895). It describes weakly nonlinear and dispersive progressive unidirectional free-surface waves in shallow water with constant depth (Peregrine, 1983). The solutions of the KdV are stable, in that the wave amplitude does not alter significantly when the initial wave train is perturbed. This is the mathematical explanation of why rogue waves in shallow water cannot be a result of the modulational instability. The inverse scattering transform (IST) was introduced as a tool to solve the KdV equation (Gardner et al., 1967), and later-on also a broader range of evolution equations (Ablowitz et al., 1974). The name scattering transform 
has its roots in physics, where the tools applied in the derivation of the IST are used to analyse how particles behave in the interaction with a scatterer (Wahls and Poor, 2015). When a time series is close to linear, its scattering data essentially reduces to the linear Fourier Transform (FT). Therefore, the IST has been called a "natural extension of Fourier analysis to nonlinear problems" (Ablowitz et al., 1974). Henceforth in this paper, the method is referred to as the nonlinear Fourier transform for the KdV equation (KdV-NLFT). Zabusky and Kruskal (1965) discussed, by numerically solving the KdV equation, the decomposition of an initial signal into a train of solitons. Brühl and Oumeraci (2016) confirmed in laboratory experiments and numerical simulations that long cosine waves in shallow water decompose into trains of solitons that are solutions to the $\mathrm{KdV}$ equation and that show larger amplitudes than the initial wave height. The KdV-NLFT yields a discrete set of eigenvalues and a continuous spectrum. Each of the eigenvalues corresponds to a soliton (Peregrine, 1983), and the continuous spectrum to oscillatory waves. The asymptotic development of the solution with time leads to a decay of the oscillatory part and the solitons asymptotically dominate the solution (Zabusky and Kruskal, 1965).

The nonlinear interaction of solitons in shallow water has been discussed with regard to its role in rogue wave generation. Based on the KdV-NLFT, Pelinovsky et al. (2000) showed that dispersive focusing is possible in the nonlinear case, given the nonlinear wave train includes at least one soliton. Equivalently to the linear case, in which rogue waves evolve from the superposition of wave components, nonlinear focusing is then the interaction between one or multiple solitons with oscillatory waves, due to their velocity difference. For the unidirectional case, several authors (Kharif and Pelinovsky, 2003; Soomere and Engelbrecht, 2005) found that the interaction of KdV solitons does not lead to a significant increase in surface elevation. Soomere (2010) considered that since soliton interaction in the unidirectional case does not lead to an enhancement in surface elevation, a higher nonlinearity should even lead to a decrease in rogue wave occurrence probability. Since this is not consistent with observations, he concluded that directionality must play a role for the rogue wave generation in shallow water. Indeed, crossing solitons are known to be able to produce large amplitudes (Peterson et al., 2003). Zakharov and Shabat (1975) found the analytical two-soliton solution of the Kadomtsev-Petviashvili (KP) equation describing this case. Hammack et al. (1989) investigated two long-crested solitary waves propagating in different directions and interacting. In contrast to linear superposition, the interaction of two crossing solitons may produce a crest up to four times higher than the incoming waves (Peterson et al., 2003). Peterson et al. (2003) discussed the interaction of shallow-water solitons against the background of heavy fast ferry traffic. They made this restriction because shallow-water areas with heavy ship traffic are more likely to produce regular, long-crested 2D wave trains, necessary for their model of rogue waves, than wind sea on the open ocean. They emphasised that the interaction area is restricted and it is unlikely to detect an interaction soliton in one-point in-situ measurements. Osborne et al. (1991) analysed nearly unidirectional shallow-water measurement data from the Adriatic Sea in the framework of the $\mathrm{KdV}$ equation for an IST with quasi-periodic boundary conditions. They found several solitons in the discrete spectrum of the IST and pointed out their physical relevance for the structure of the time series. Since rogue wave occurrence in shallow water that goes beyond second order has not been sufficiently explained, and almost all investigations in previous work are based on theoretical considerations, numerical simulations or laboratory experiments, we consider real measurement time series in the framework of the $\mathrm{KdV}$ equation. We expand the investigation of data measured by a surface-following buoy off the coast of Norderney in the southern North Sea, for which second-order distributions have been shown to underestimate rogue wave 
occurrence (Teutsch et al., 2020). We obtain a discrete soliton spectrum from the nonlinear Fourier transform for the KdV equation with vanishing boundary conditions (vKdV-NLFT) and explore to what extent the presence of solitons might contribute to this enhanced statistical rogue wave occurrence. For this purpose, we compare the soliton spectra of samples with and samples without rogue waves. The paper is structured as follows. Section 2.1 describes the measurement site and the dataset and gives a definition for rogue waves. In Sect. 2.2, the application of vKdV-NLFT to the measurement data is explained. Sect. 3 consists of two parts. In Sect. 3.1, we explore the direct association of solitons calculated from NLFT with rogue waves, while Sect. 3.2 discusses statistical differences in the soliton spectra of time series with and without rogue waves. In Sect. 4, we discuss the time windows and location for which our results are valid, and suggest further investigations. In Sect. 5 our conclusions are presented.

\section{Methods}

\subsection{Measurement site and dataset}

We analysed wave elevation data measured by a surface-following buoy off the coast of the island Norderney in the German Bight in the time period between 2011 and 2016. The measurement buoy was deployed at a nominal water depth of $h=10 \mathrm{~m}$, which was assumed to be constant for the following analyses. Actually, the water depth off the coast of Norderney is not constant, as the bathymetry at the location is spatially highly variable with strong gradients (Fig. 2). The buoy is located right above a steep slope, running perpendicular to the mean incoming wave direction (Fig. 3). Since the buoy is restricted only by its mooring, it has the possibility to move horizontally. The actual water depth $h$ below the horizontally moving buoy may then be subject to rapid changes. In addition, the tidal range at the site is about $2.5 \mathrm{~m}$ (NLWKN, 2021), which further causes the water depth to vary. 


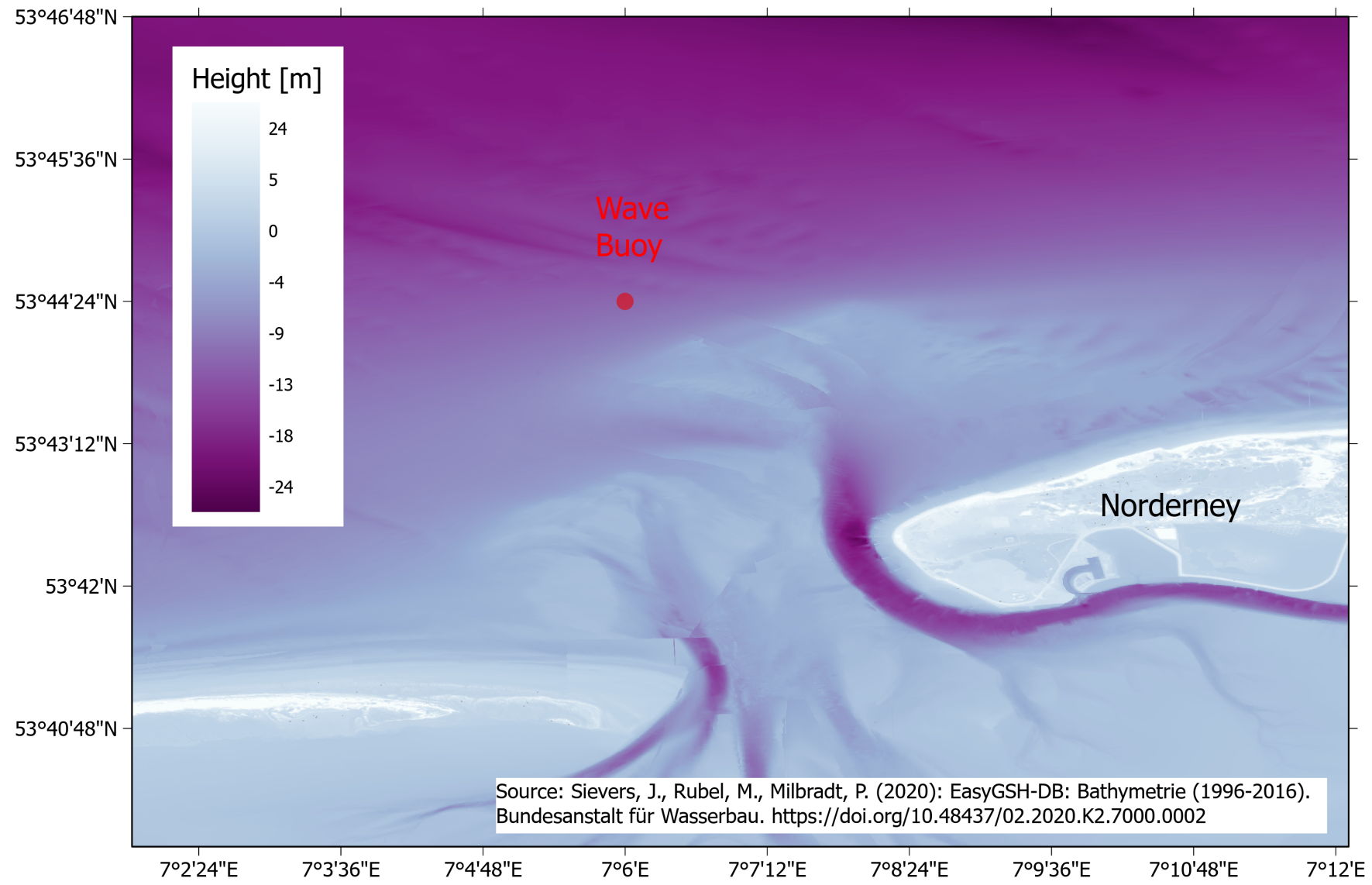

Figure 2. Bathymetry conditions $[\mathrm{NN}+\mathrm{m}]$ at Norderney and the position of the measurement buoy. 


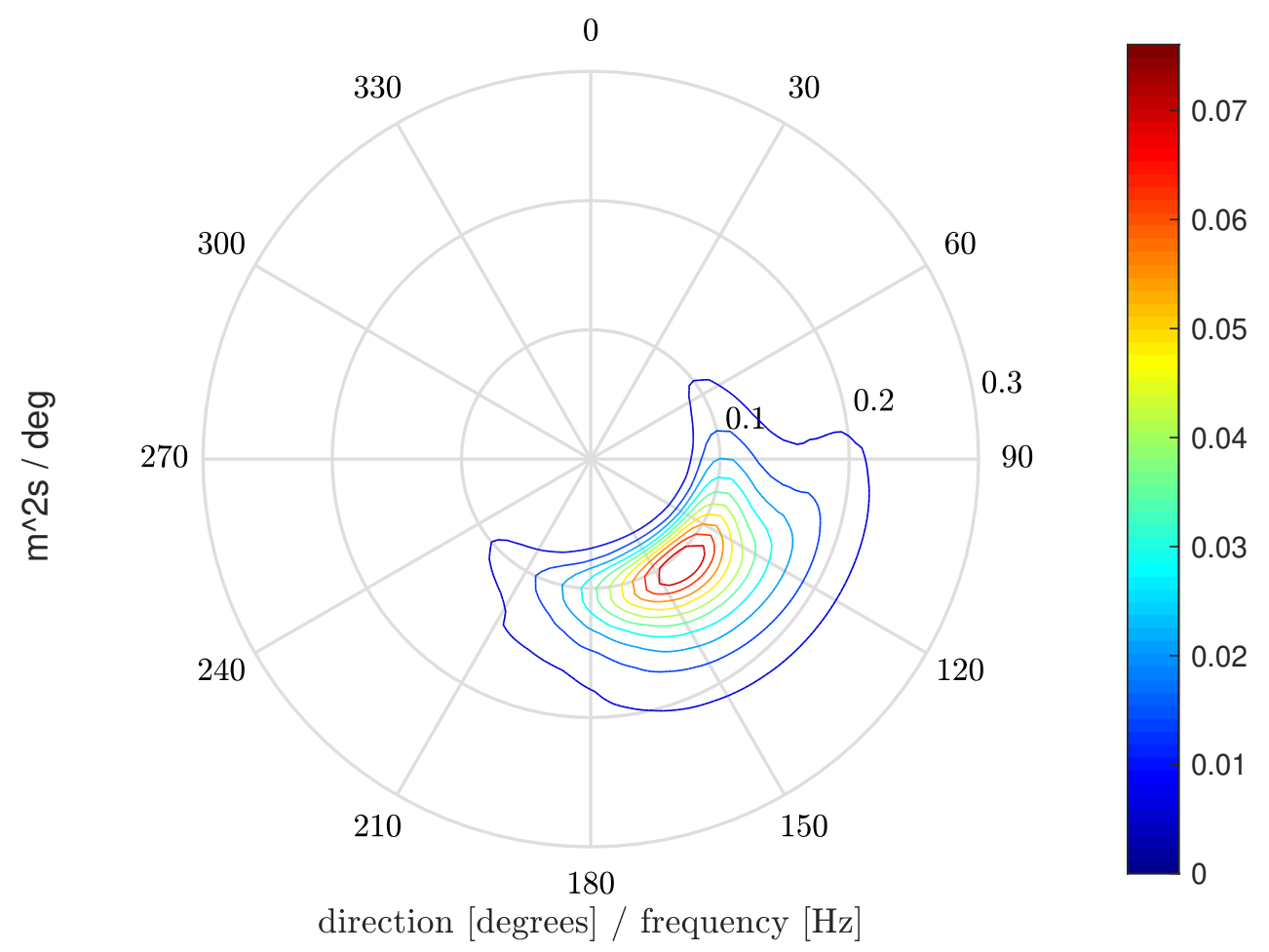

Figure 3. Mean directional wave spectrum from the time period 2011-2016, obtained by use of DIWASP (Johnson, 2002).

The wave data were measured at a frequency of $1.28 \mathrm{~Hz}$ and are available as a set of time series (samples) of 30 minute length. To exclude low-energy sea states in the following, only samples with a significant wave height $H_{s}$ above the long-term 70th percentile of the significant wave height, $H_{s, 70}=1.29 \mathrm{~m}$, were included in the analysis. The significant wave height $H_{s}$ is here defined as the mean of the highest $30 \%$ of the wave heights in a 30 minute sample. $H_{s, 70}$ was calculated from the significant wave heights $H_{s}$ of all 30 minute samples during the six years of available measurement data. On the one hand, this excludes possible measurement uncertainties caused by small waves that are only described by a few points, and on the other hand, it includes only rogue waves of heights relevant for offshore activities. Since the KdV equation for shallow water was to be applied to the data, only samples satisfying shallow-water conditions were included in the study. The shallow-water condition used was

$\frac{h}{L}<0.22$

with water depth $h$ and wavelength $L$. The wavelength was calculated as

$L=T_{p} * c$ 
from the peak period $T_{p}=1 f_{p}^{-1}$ of each sample, with $f_{p}$ the peak frequency in the linear fast Fourier transform (FFT) spectrum of the sample, and the shallow-water wave celerity $c=\sqrt{g h}$ with gravity $g$. Following Eq. (1) and Eq. (2), the condition for the peak period may be written as

$T_{p}>\frac{h}{0.22 \cdot c}$.

For a water depth of $h=10 \mathrm{~m}$, the peak period thus had to satisfy the condition $T_{p}>4.6 \mathrm{~s}$, in order for a sample to classify for shallow-water conditions. We based the shallow-water condition on the peak period $T_{p}$ of the entire sample to assume that shallow-water wave properties as described by the $\mathrm{KdV}$ equation strongly contribute to the wave processes in the sample. Nevertheless, it was additionally ensured that each of the individual rogue waves (or the highest wave in each sample that did not contain a rogue wave) satisfied shallow-water conditions, based on its period $T_{\max }$. Of all the selected samples above $H_{s, 70}$, shallow-water conditions applied in more than $98 \%$ of the cases and were thus the dominant condition in these samples. The $2 \%$ of the samples not satisfying shallow-water conditions were discarded and not considered in the analysis.

Rogue waves are commonly defined as waves with an individual height $H$ from crest to trough of (Haver and Andersen, 2000)

$H \geq 2.0 H_{s}$

and/or waves with a crest height $C$ above still water level of (Haver and Andersen, 2000)

$C \geq 1.25 H_{s}$

In a previous study based on measurement data from the southern North Sea (Teutsch et al., 2020), we found that the rogue wave frequency significantly deviated from the Forristall distribution for wave heights larger than $2.3 \mathrm{H}_{s}$. Therefore, in the present study we further define "extreme rogue waves" by a more strict height criterion of

$H \geq 2.3 H_{s}$.

For the definition of a wave, the zero-upcrossing method was used.

The measured time series were subdivided into five categories:

"normal samples"- measurement samples that did not include any rogue wave.

"height rogue samples"- measurement samples that include a rogue wave only according to the height criterion defined in Eq. (4), while excluding the extreme rogue waves according to Eq. (6) and excluding the double rogue samples (see below).

"crest rogue samples"- measurement samples that included a rogue wave only according to the crest criterion defined in Eq. (5), while excluding the double rogue samples.

"double rogue samples"- measurement samples that included a rogue wave according to both the criteria defined in Eq. (4) and Eq. (5), while excluding the extreme rogue waves according to Eq. (6). 
https://doi.org/10.5194/nhess-2022-28

Preprint. Discussion started: 4 March 2022

(c) Author(s) 2022. CC BY 4.0 License.

(c) (i)

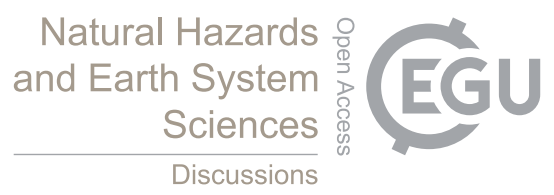

"extreme rogue samples"- measurement samples that included a rogue wave according to the height criterion defined in Eq. (6), while excluding the double rogue samples.

Examples of each time series category are shown in Fig. 4. Table 1 shows the number of samples and its percentage in each category. 


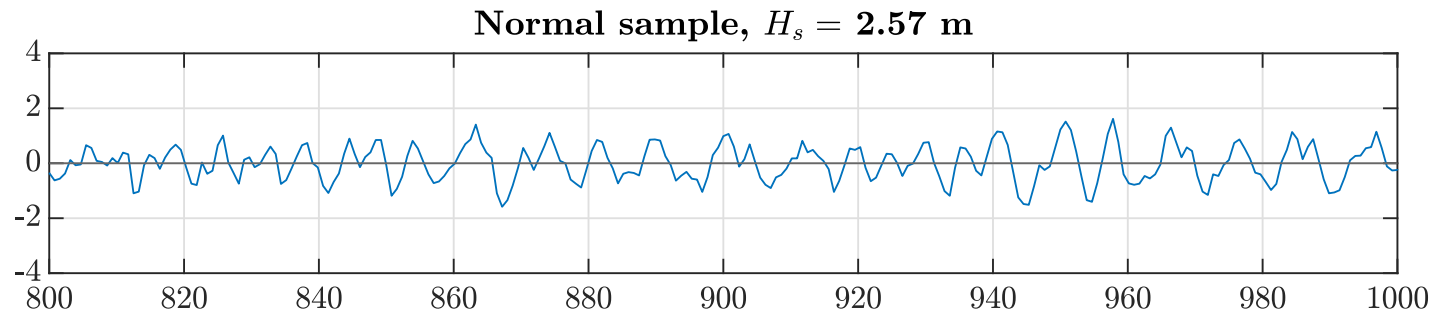

Height rogue sample, $H_{s}=\mathbf{2 . 3 7} \mathbf{m}$

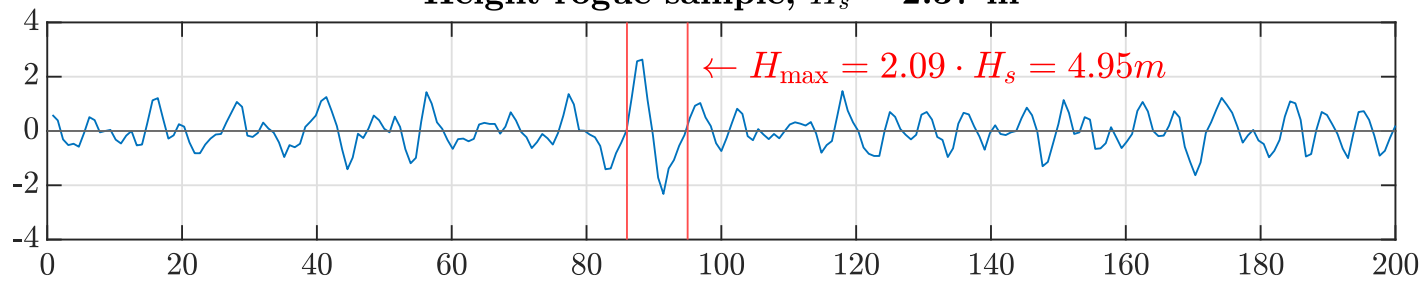

Crest rogue sample, $H_{s}=2.40 \mathrm{~m}$

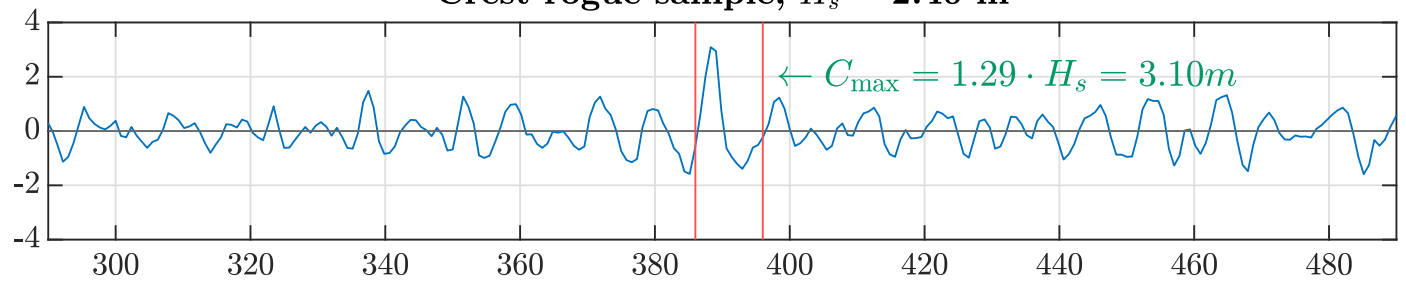

Double rogue sample, $H_{s}=\mathbf{2 . 4 2} \mathrm{m}$

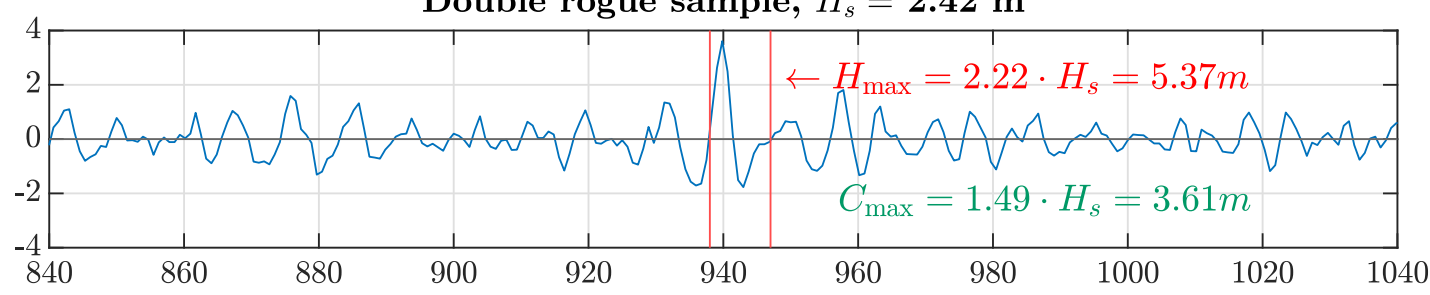

Extreme rogue sample, $H_{s}=3.21 \mathrm{~m}$

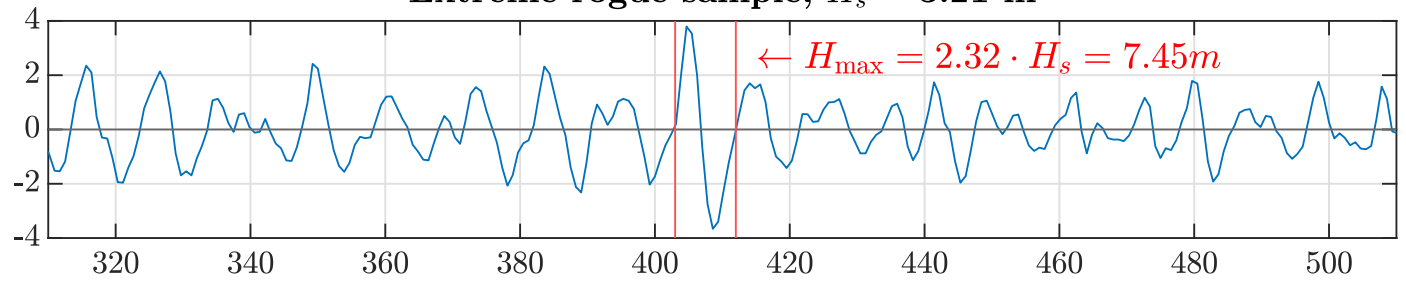

Figure 4. $200 \mathrm{~s}$ sections taken from example time series illustrating rogue waves for each of the four rogue wave categories, and a normal sample with a similar value of $H_{s}$ for comparison. Vertical red lines mark the two zero-crossings of the rogue wave. Rogue wave/crest heights are indicated in red/green. 
Table 1. Positions and water depths of the measurement sites.

\begin{tabular}{ccccccc}
\hline Sample category & Normal & Height rogue & Crest rogue & Double rogue & Extreme rogue & Total \\
\hline No. of samples & 13.984 & 833 & 95 & 151 & 93 & 15.156 \\
Percentage & $92.3 \%$ & $5.5 \%$ & $0.6 \%$ & $1.0 \%$ & $0.6 \%$ & $100 \%$ \\
\hline
\end{tabular}

\subsection{Application of the Korteweg-de Vries equation with vanishing boundary conditions to the measurement data}

A vKdV-NLFT was applied to the data, to obtain the discrete soliton spectrum of each time series. The KdV equation was introduced by Korteweg and de Vries (1895). It describes the evolution of weakly nonlinear and dispersive progressive unidirectional free-surface waves in shallow water $\left(h L^{-1}<0.22\right)$ with constant depth. For the analysis of space series (fixed at one point in time), the space-like KdV equation (sKdV) is given e.g. in Osborne (2010), with reference to Korteweg and de Vries (1895) as

$u_{t}+c u_{x}+\alpha u u_{x}+\beta u_{x x x}=0$,

in which $u=u(x, t)$ is a free-surface space series, developing in space $x$ and time $t$. The subscripts $x$ and $t$ denote partial derivatives, $c$ is the phase speed in shallow water, $\alpha=(3 c)(2 h)^{-1}$ and $\beta=\left(c h^{2}\right) / 6$ are constants, depending on the phase speed $c$ and the water depth $h$. Equation (7) can be adapted to the analysis of time series (fixed at one point in space, like e.g. buoy measurements. For the case of a free-surface elevation time series $u\left(x_{0}, t\right)$ (see f.ex. Fig. 5) at base point $x_{0}$, it is then described by the time-like KdV equation (tKdV) (Osborne, 1993)

$u_{x}+c^{\prime} \cdot u_{t}+\alpha^{\prime}+u \cdot u_{t}+\beta^{\prime} \cdot u_{t t t}=0$,

in which $c^{\prime}=c^{-1}=(\sqrt{g h})^{-1}, \alpha^{\prime}=-\alpha\left(c^{2}\right)^{-1}$ and $\beta^{\prime}=-\beta\left(c^{4}\right)^{-1}$. For our application of the KdV-NLFT, we assumed initial conditions with vanishing boundaries

$\lim _{t \rightarrow \pm \infty} u\left(x_{0}, t\right)=0$

sufficiently fast. Since we were mainly interested in the soliton part of the nonlinear spectrum and solitons are not periodic, we preferred vanishing (vKdV-NLFT) to periodic (pKdV-NLFT) boundary conditions. In the KdV-NLFT, solitons are easily identified as the discrete part of the nonlinear spectrum. We applied the vKdV-NLFT by using the MATLAB (2019) interface to the software library FNFT (Wahls et al., 2018), development version (commit 681191c). Its solution consists of a discrete soliton spectrum and a continuous spectrum representing oscillatory waves. Figure 5 shows an example of a measurement time series and its corresponding soliton spectrum. To distinguish them from oscillatory waves, solitons are displayed on a negative frequency axis. Technically, the frequency axis has no physical meaning, because a soliton, for which the surface elevation does not cross the still water level, has no frequency (Brühl and Oumeraci, 2016). However, from the soliton solution of the $\mathrm{tKdV}$, an angular frequency is obtained as

$\Omega=2 \pi \cdot F=\sqrt{\frac{3 A g}{4 h^{2}}}$. 
https://doi.org/10.5194/nhess-2022-28

Preprint. Discussion started: 4 March 2022

(c) Author(s) 2022. CC BY 4.0 License.

215 Since this equation relates the frequency $F$ to the amplitude $A$ of the soliton, the frequency sorts the solitons in the spectrum by their amplitude. The vKdV-NLFT was applied to all 15.156 samples listed in Table 1.
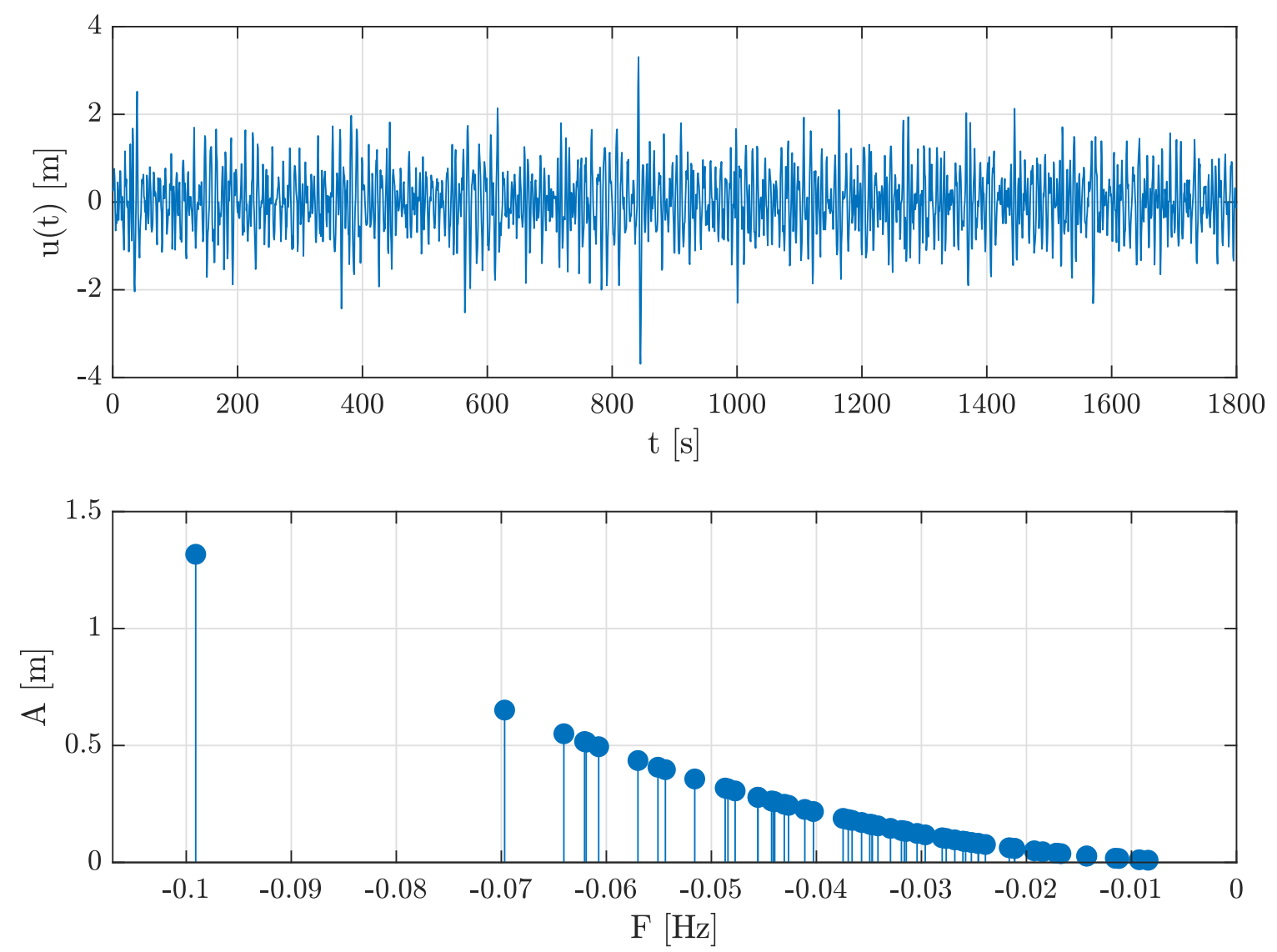

Figure 5. Example of a time series including a rogue wave at approx. $820 \mathrm{~s}$, and its corresponding soliton spectrum, calculated from vKdVNLFT. The time series with $H_{\max } H_{s}^{-1}=2.58, H_{\max }=7.00 \mathrm{~m}$ and $H_{s}=2.71 \mathrm{~m}$, was measured on 17 October 2013, starting at 11:30.

Solitons were found in all samples, with and without rogue waves. The aim of the study was to explore the role of the determined solitons for the generation of rogue waves. In the first part of the study, it was investigated whether specific solitons in the NLFT spectrum could be associated with the recorded rogue waves. For this purpose, all free-surface elevations between the two zero-crossings of a rogue wave (or largest wave, for normal samples) were scaled down to 80 \% (Fig. 6). The KdVNLFT was then repeated for the modified time series, which resulted in a new soliton spectrum. It was monitored which of the solitons had changed in amplitude A (and, therefore, in frequency F), due to the change in wave height. These solitons were assumed to have the same position as the rogue/ maximum wave. In the second part of the study, we explored whether 
https://doi.org/10.5194/nhess-2022-28

Preprint. Discussion started: 4 March 2022

(C) Author(s) 2022. CC BY 4.0 License.

(c) (i)

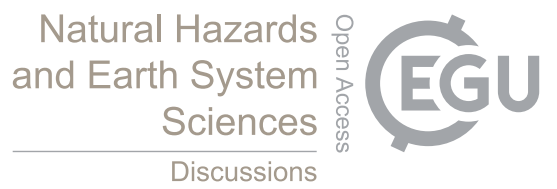

the spectra calculated from rogue wave time series showed differences when compared to those calculated from normal time series.

\section{Results}

\subsection{Attribution of solitons to rogue waves}

Solitons were attributed to specific rogue waves, following the procedure described in Sect. 2.2. We found in each case that the amplitude of one large soliton significantly decreased for a reduced rogue wave (or maximum wave) height. Also in the group of smaller solitons, slight changes in amplitudes were observed. Since for solitons, amplitude A and frequency F are related according to Eq. (10), the reduction in amplitude corresponded to a simultaneous shift in frequency, which can be seen in the soliton spectrum (Fig. 6). The reduced solitons can be regarded to be associated with the rogue wave in the time series, while the other solitons in the spectrum maintained their amplitudes. The solitons with constant amplitudes can be regarded not to be associated with the rogue wave. We refer to the amplitudes of the $n=1 \ldots i$ solitons associated with the rogue wave as $A_{S}^{i}$, with $A_{S}^{1}$ denoting the largest attributed soliton. Although often the case, the largest soliton attributed to the rogue wave was not necessarily the largest soliton in the spectrum (Fig. 7). 
(a) Time series

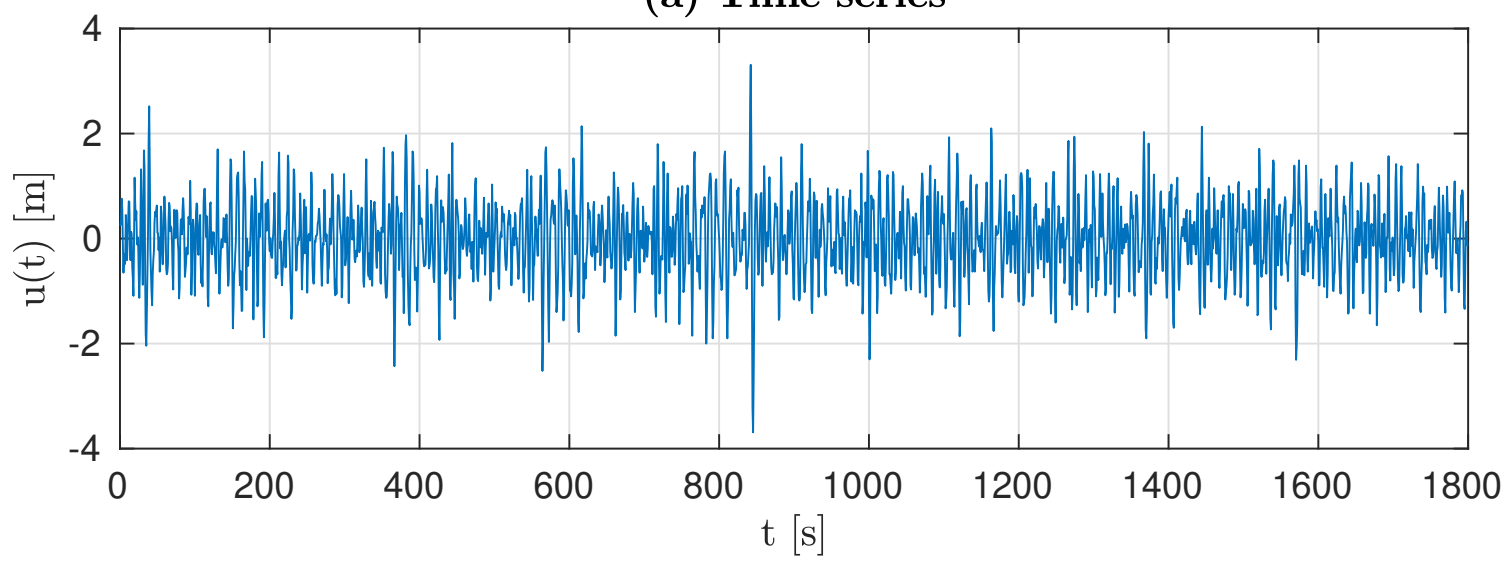

(b) Zoomed-in time series

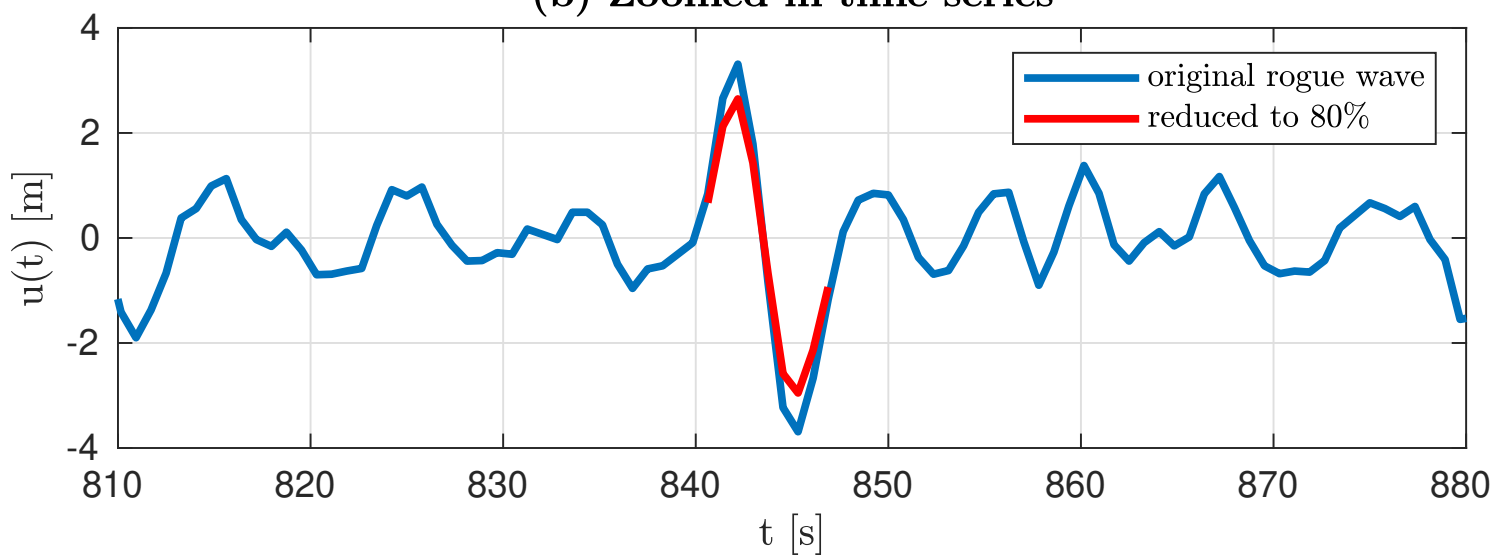

(c) Soliton spectrum

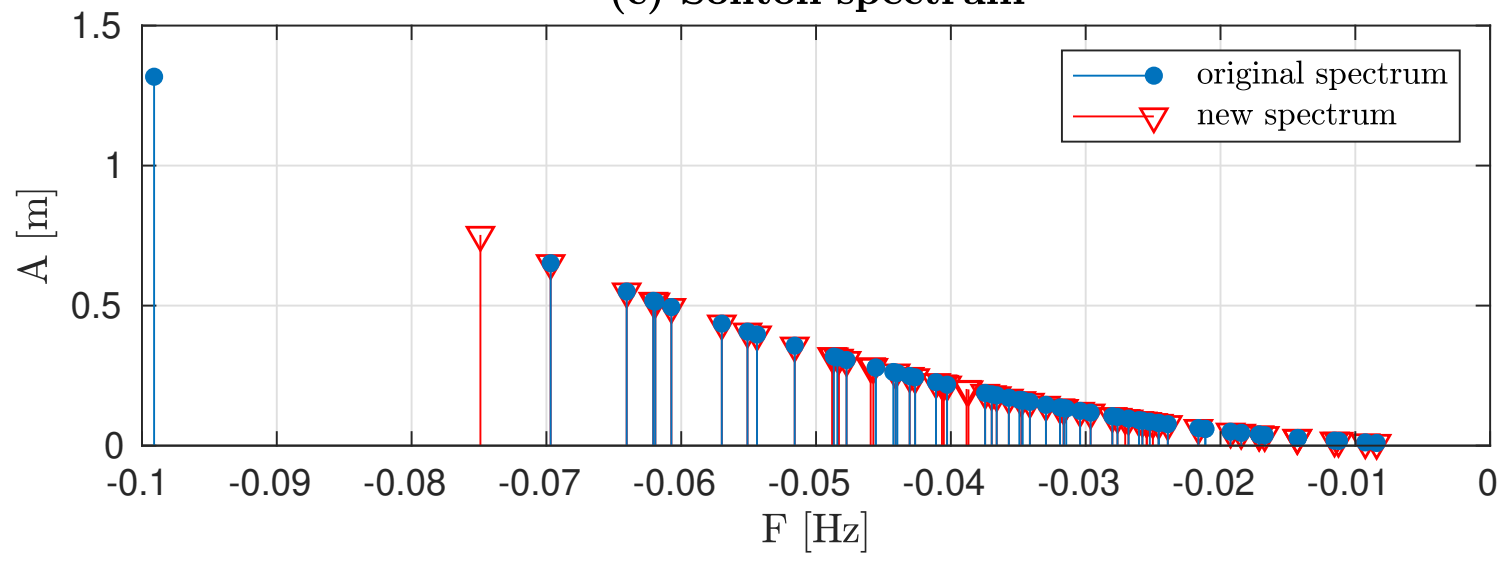

Figure 6. From top to bottom: (a) extreme rogue wave time series from 17 October 2013, starting at 11:30; (b) magnified view of the rogue wave (blue curve) and reduction of its elevation to $80 \%$ (red curve); (c) soliton spectra of the original (blue circles) and the modified time series (red triangles), resulting from vKdV-NLFT. 
(a) Time series

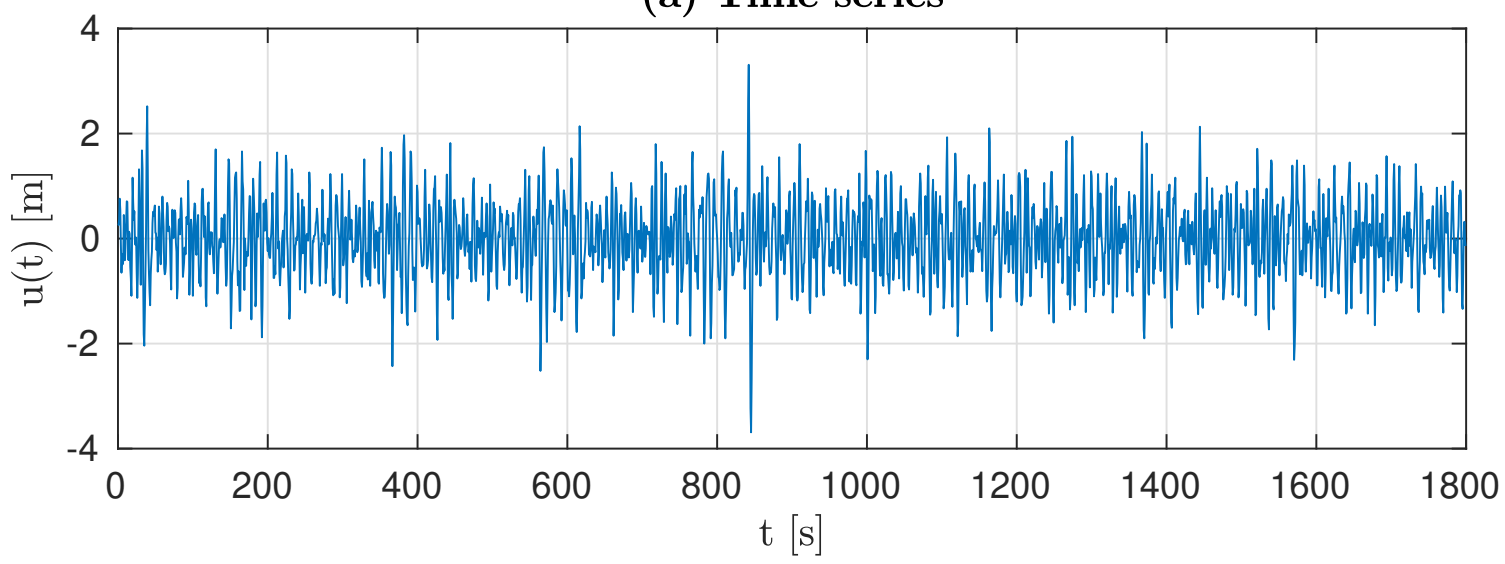

(b) Zoomed-in time series

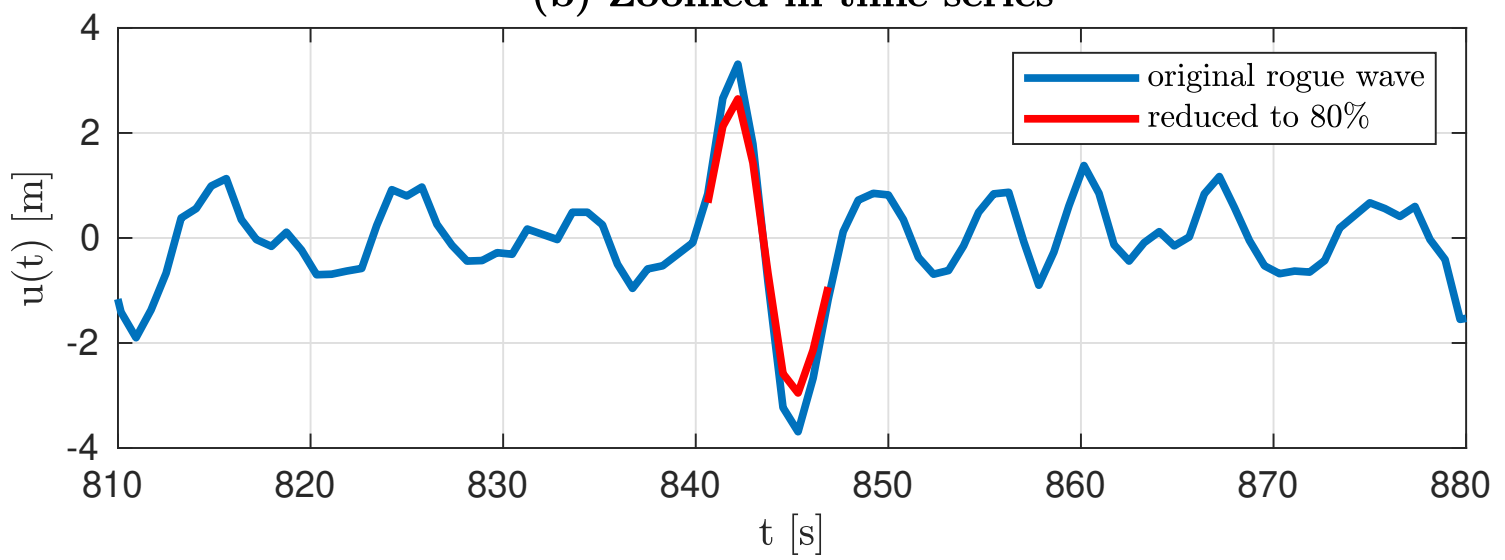

(c) Soliton spectrum

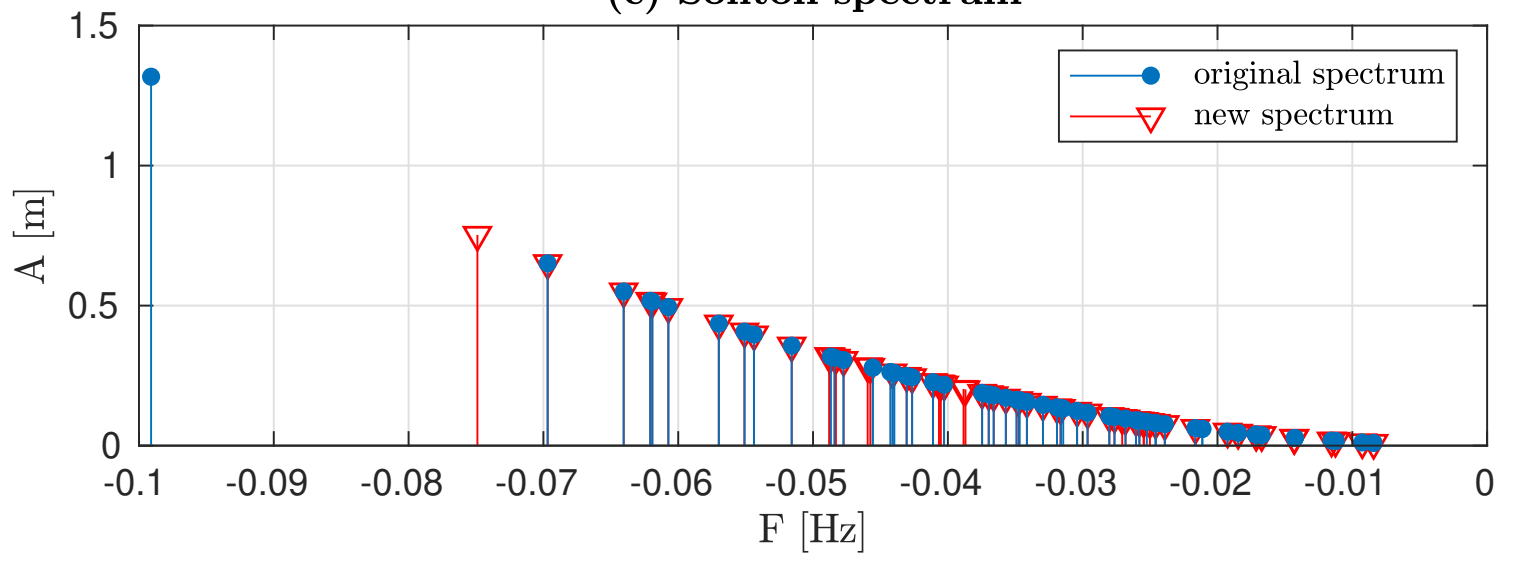

Figure 7. From top to bottom: (a) double rogue wave time series from 27 April 2016, starting at 20:30; (b) magnified view of the rogue wave (blue curve) and reduction of its elevation to $80 \%$ (red curve); (c) soliton spectra of the original (blue circles) and the modified time series (red triangles), resulting from vKdV-NLFT. 
We extracted the amplitude of the largest attributed soliton $A_{S}^{1}$ for each time series and compared it to the rogue wave height $H$ (for rogue waves according to any of the two height criteria, including double rogue waves, Fig. 8(a)) or the crest height $C$ of the rogue wave (for rogue waves according to the crest criterion, including double rogue waves, Fig. 8(b)). A comparison of the soliton amplitude $A_{S}^{1}$ to the largest wave height $H_{\max }$ and the largest crest height $C_{\max }$ in normal samples has been added for reference (Fig. 8(c) and (d)). The gradients of the linear regression curves express increasing $A_{S}^{1}$ with increasing $H H_{\max }^{-1}$ and $C H_{\max }^{-1}$. The scatter of the data suggests an upper limit of $A_{S}^{1}$ between $2 \mathrm{~m}$ and $3 \mathrm{~m}$. The goodness of fit of each curve to the data is given in terms of the coefficient of determination

$R^{2}=\frac{S S_{\text {res }}}{S S_{\text {total }}}$

245 in which $S S_{\text {res }}$ is the sum of squares of residuals with respect to the regression curve, and $S S_{\text {total }}$ is the sum of squared residuals with respect to the average value of the data and thus a measure of the variance. $R^{2}$ indicates that the linear curves fit the results from height and extreme rogue wave samples better than the results from normal, double and crest rogue samples. $R^{2}$ is higher in Fig. 8(a) than in Fig. 8(b)-(d).

Moreover, it is seen that the amplitude of the largest soliton is always smaller than the rogue wave crest/ height itself. This is in agreement with results by Osborne et al. (1991), who identified solitons in measurement data from the Adriatic sea by applying the NLFT with quasi-periodic boundary conditions to the KdV equation. Thus, the mere existence of a soliton is not sufficient to explain the presence of a rogue wave in our data. Our investigation revealed that in all cases some smaller solitons were additionally associated with a rogue wave. Typical values of the amplitude of the second-largest soliton $A_{S}^{2}$ are $20-30 \%$ of $A_{S}^{1}$. The amplitude of the third-largest attributed soliton $A_{S}^{3}$ is typically 10-20\% of $A_{S}^{1}$. The interaction of unidirectional solitons, however, as described by KdV, is known not to result in exceptional increases in wave elevation(Kharif and Pelinovsky, 2003). Hence, the soliton spectrum alone does not yield a satisfactory explanation of the generation mechanism of extreme waves/ crests. One may speculate that the formation of the rogue wave in these cases is a result of the interaction of one or several solitons with the underlying oscillating wave field, a hypothesis which will need further analyses to be validated. 
https://doi.org/10.5194/nhess-2022-28

Preprint. Discussion started: 4 March 2022

(c) Author(s) 2022. CC BY 4.0 License.

(c) (1)
Natural Hazards and Earth System Sciences

Discussions (a)

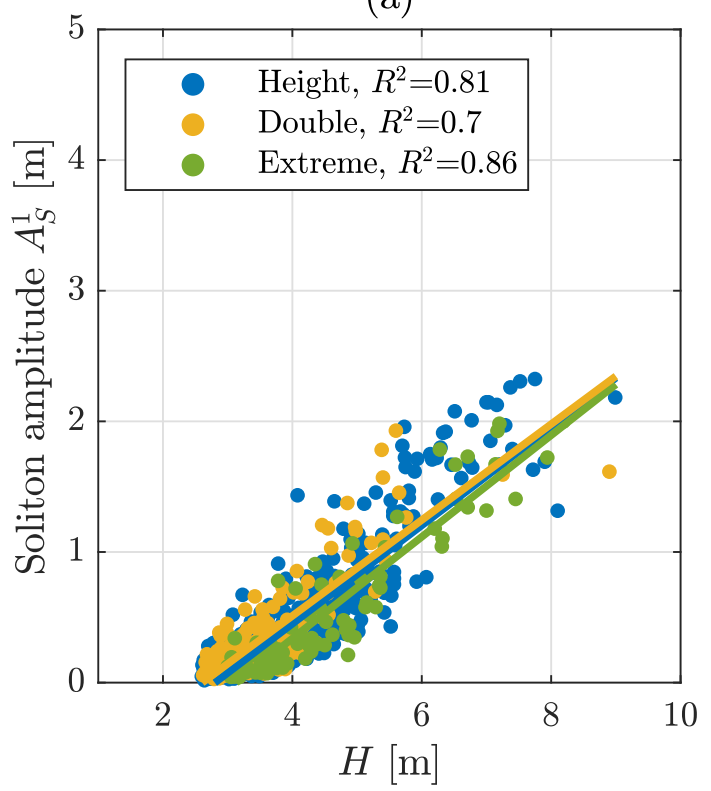

(c)

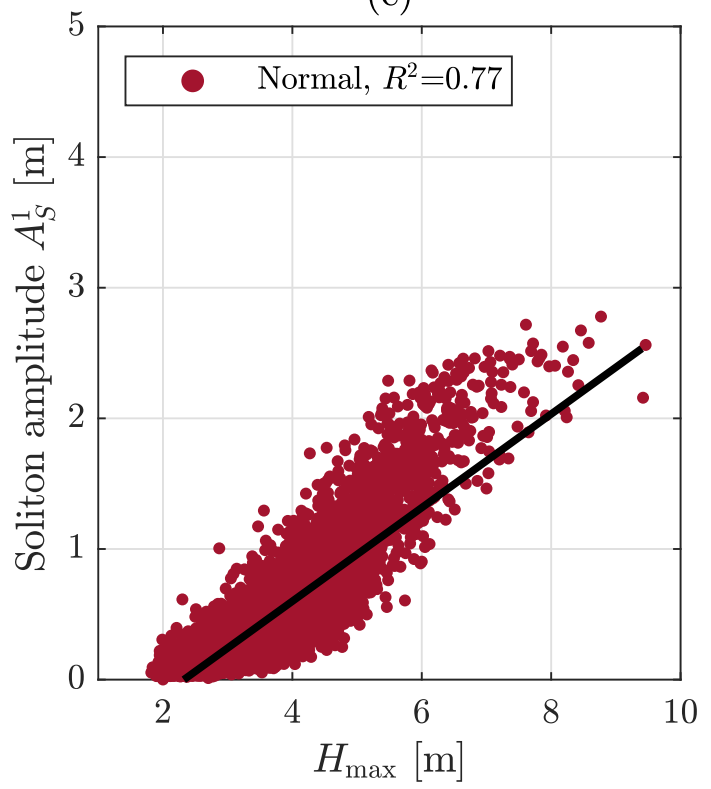

(b)

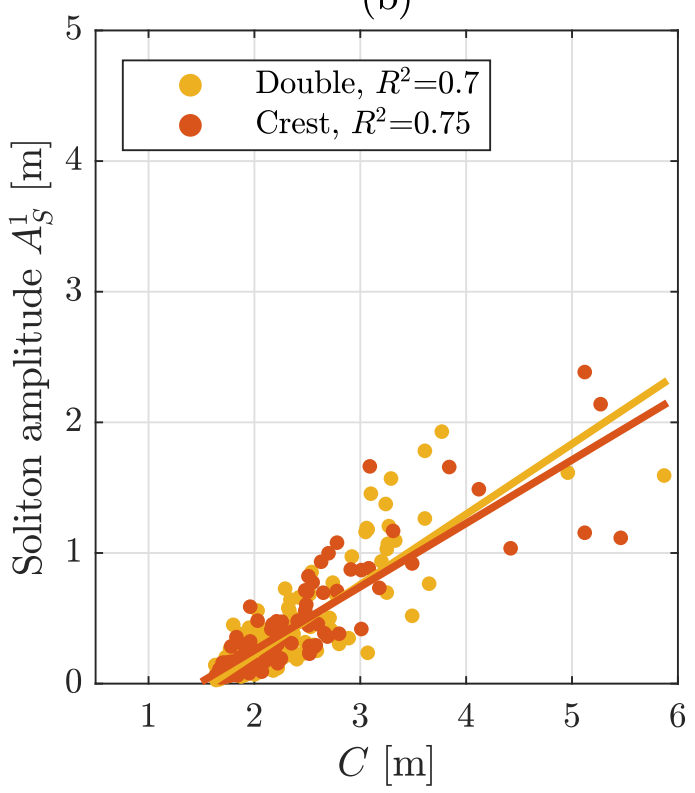

(d)

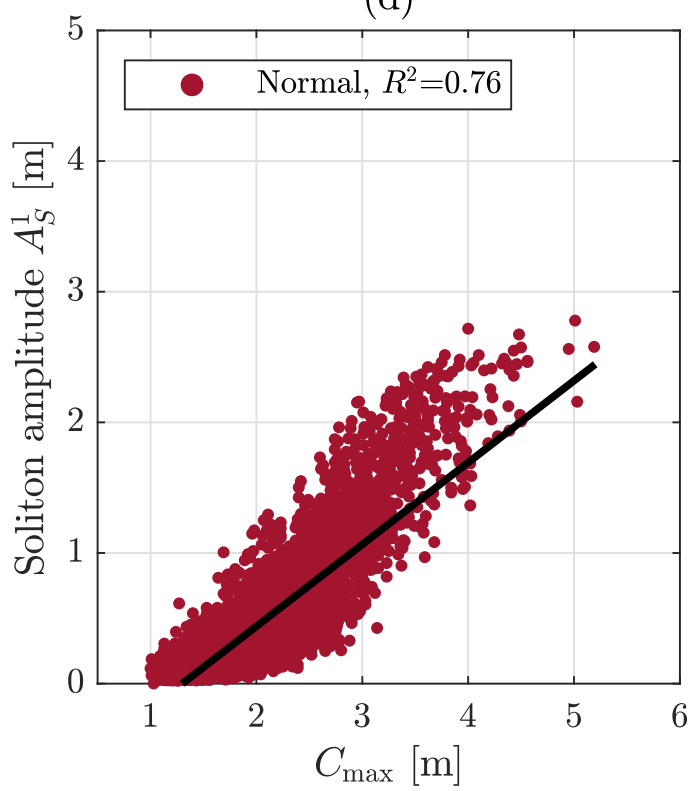

Figure 8. Amplitude of the largest soliton attributed to the highest wave, $A_{S}^{1}$, in the time series for normal samples (upper row) or the rogue wave (lower row) as a function of maximum wave height (left column) or maximum crest height (right column). The goodness of fit of the linear regression curves is given in terms of $R^{2}$. 
So far, the results show that high soliton amplitudes in the spectrum are associated with high absolute values of wave heights or crests. However, this does not necessarily imply that high solitons play a role in forming individual waves that are exceptional with respect to the surrounding wave field. To remove the influence of the underlying sea state, the soliton amplitudes $A_{S}^{1}$ were normalised by the significant wave height $H_{s}$ of the corresponding sample. By relating the normalised soliton amplitudes to the different time series categories, the importance of solitons for the relative height of rogue or maximum waves was investigated (Fig. 9). If solitons are to play a major role for the presence of rogue waves, their normalised amplitudes are expected to increase from normal samples with $H\left(H_{s}\right)^{-1}<2.0$ through height and double rogue waves $\left(2.0 \leq H\left(H_{s}\right)^{-1}<2.3\right)$ to extreme rogue waves $\left(H\left(H_{s}\right)^{-1} \geq 2.3\right)$. In fact, the median values of $A_{S}^{1}\left(H_{s}\right)^{-1}$ are higher for rogue wave samples than for normal samples, meaning the distributions calculated from the rogue wave samples are shifted to the right with respect to the distribution calculated from normal samples (Fig. 9). Additionally, the rogue wave sample distributions, and especially those calculated from crest and extreme rogue samples, show heavier right tails. The differences in the distributions suggest that solitons play a role in rogue wave generation. It is striking that not only extreme rogue waves, but also crest rogue waves had a tendency to be associated with higher solitons. This makes sense when recalling that a soliton is not an oscillating wave and because of its shape contributes more to wave crests than to wave heights. However, although differences in normalised soliton amplitudes $A_{S}^{1}\left(H_{s}\right)^{-1}$ are present for the different categories, the distributions overlap and the positive trend with increasing relative wave height is not as pronounced as the positive trend of $A_{S}^{1}$ with increasing maximum wave height, as presented in Fig. 8. This emphasises the relevance of the considered sea state for the soliton amplitude, in that large solitons are only found in high sea states. Large solitons correspond to high wave heights $H$ and high crest heights $C$, but not necessarily to high relative wave heights $H\left(H_{s}\right)^{-1}$ or high relative crest heights $C\left(H_{s}\right)^{-1}$. Consequently, the presence of a large soliton is not sufficient to explain the presence of a rogue wave. Again, it is presumed that oscillatory wave components and/ or nonlinear interactions must contribute to the formation of rogue waves. 


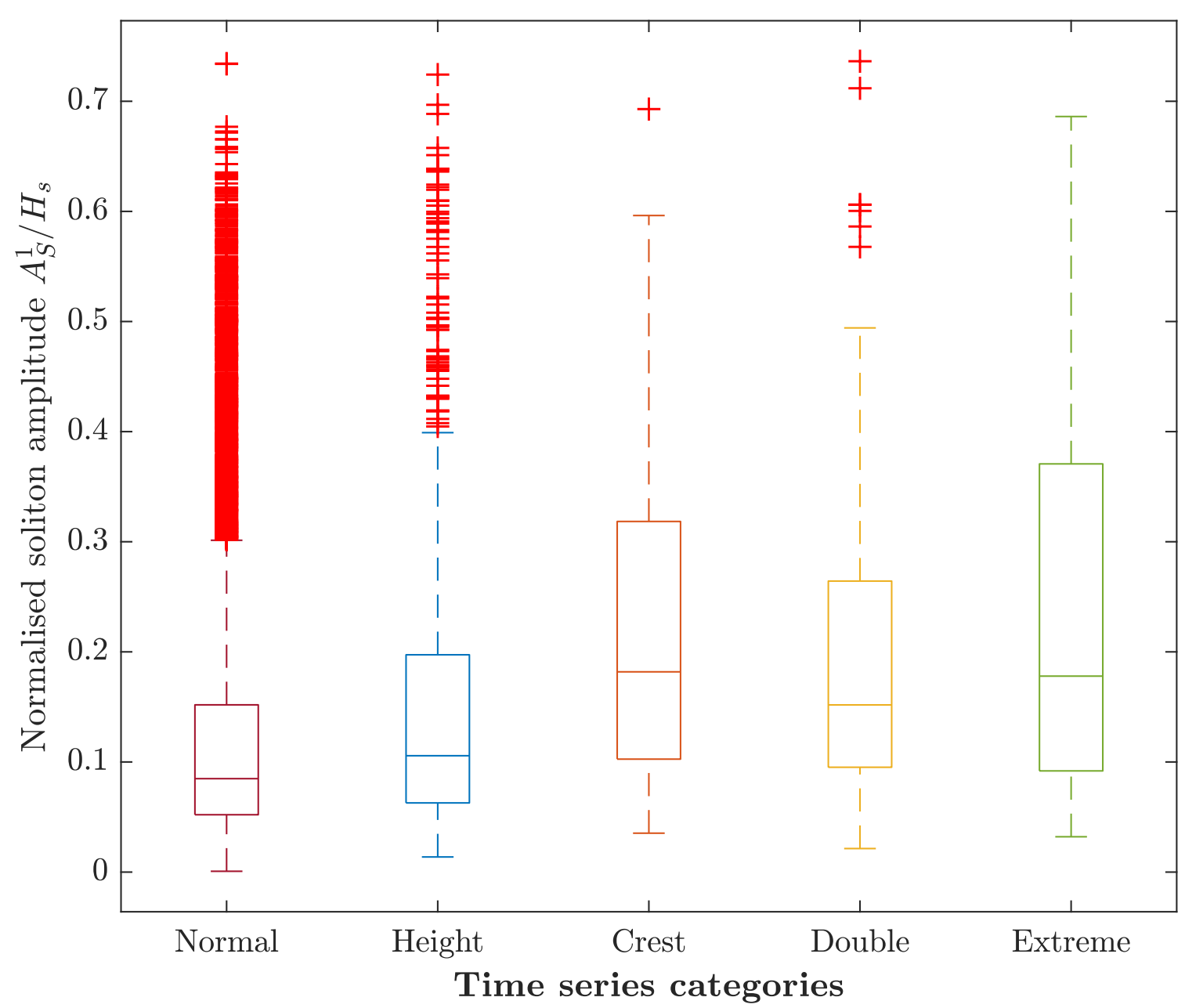

Figure 9. Amplitude of the highest soliton attributed to the rogue wave or maximum wave in the time series, normalised by the significant wave height, for the different categories of time series. Distributions are shown as box-and-whisker plots (box: interquartile range; whiskers: 1.5 times the interquartile range; horizontal line inside the box: median; red crosses: data outside the whiskers).

Since we were interested in the importance of nonlinearity in the rogue wave generation at the buoy location, we intended to quantify the nonlinearity of the rogue waves. In shallow water, the nonlinearity of waves can be described by the Ursell number (Ursell, 1953). The Ursell number in its time-like form is given, according to Osborne (2010), by

$U=\frac{3 a c^{2} T^{2}}{16 \pi^{2} h^{3}}=\frac{3}{16 \pi^{2}}\left(\frac{a}{h}\right)\left(\frac{c T}{h}\right)^{2}$,

in which we interpreted $a=C$ as the maximum elevation of the rogue wave above the still water level, following LeMéhauté (1976). The Ursell number $U$ is known to be an equivalent to the BFI for deep water waves (Slunyaev et al., 2011; Onorato 
et al., 2001) and has been used to classify wave types. It has been stated that an Ursell number of $U=0$ corresponds to linear waves, while $U=1$ points to solitary waves (Miles, 1980). In our case, the amplitudes of the largest attributed solitons show an almost linear positive trend with increasing Ursell number up until approximately $U=0.5$ (Fig. 10). Brühl (2022) classifies waves with $0.559 \leq U$ as solitary-like wave types and waves with $U<0.559$ as Airy-like, Stokes-like or cnoidal-like. For our data, in which the bulk of waves are located below $U=0.559$, this means that most rogue wave crests are not soliton-like. This is in agreement with several previous studies, which have shown that rogue waves in shallow water, despite their large amplitudes, have very small ratios of nonlinearity to dispersion (Ursell numbers), thus are almost linear (Pelinovsky et al., 2000; Kharif and Pelinovsky, 2003; Pelinovsky and Sergeeva, 2006). This again reinforces a point made earlier, that the rogue waves in our case cannot be explained by solitons alone. This may lead to the conclusion that solitons need to interact with other wave components for the formation of these rogue waves, which we have not verified. Another observation made from Fig. 10 is a threshold in soliton amplitude between $A_{S}^{1}=2.0 \mathrm{~m}$ and $A_{S}^{1}=2.8 \mathrm{~m}$, depending on the time series category, for Ursell numbers larger than approximately $U=0.5$. Referring to the classification by Brühl (2022), this implies that for the most nonlinear waves, which are those satisfying solitary wave theory, soliton amplitudes are limited. A limit in soliton height as a result of breaking is expected at amplitudes of approximately $A=8 \mathrm{~m}$ for a water depth of $h=10 \mathrm{~m}$, as the breaking criterion for solitary waves is $A h^{-1}=0.78$ (McCowan, 1891) or $A h^{-1}=0.83$ (Lenau, 1966). Therefore, shallow-water wave breaking at the location of the buoy can be excluded. The reason for the limit in soliton amplitude already at $A_{S}^{1}=2.5 \mathrm{~m}$ to $A_{S}^{1}=3 \mathrm{~m}$ could be limited energy input by wind (see Middleton and Mellen (1985) for soliton generation by wind), or a shoal in front of the measurement buoy causing the larger waves to break before they reach the buoy. 

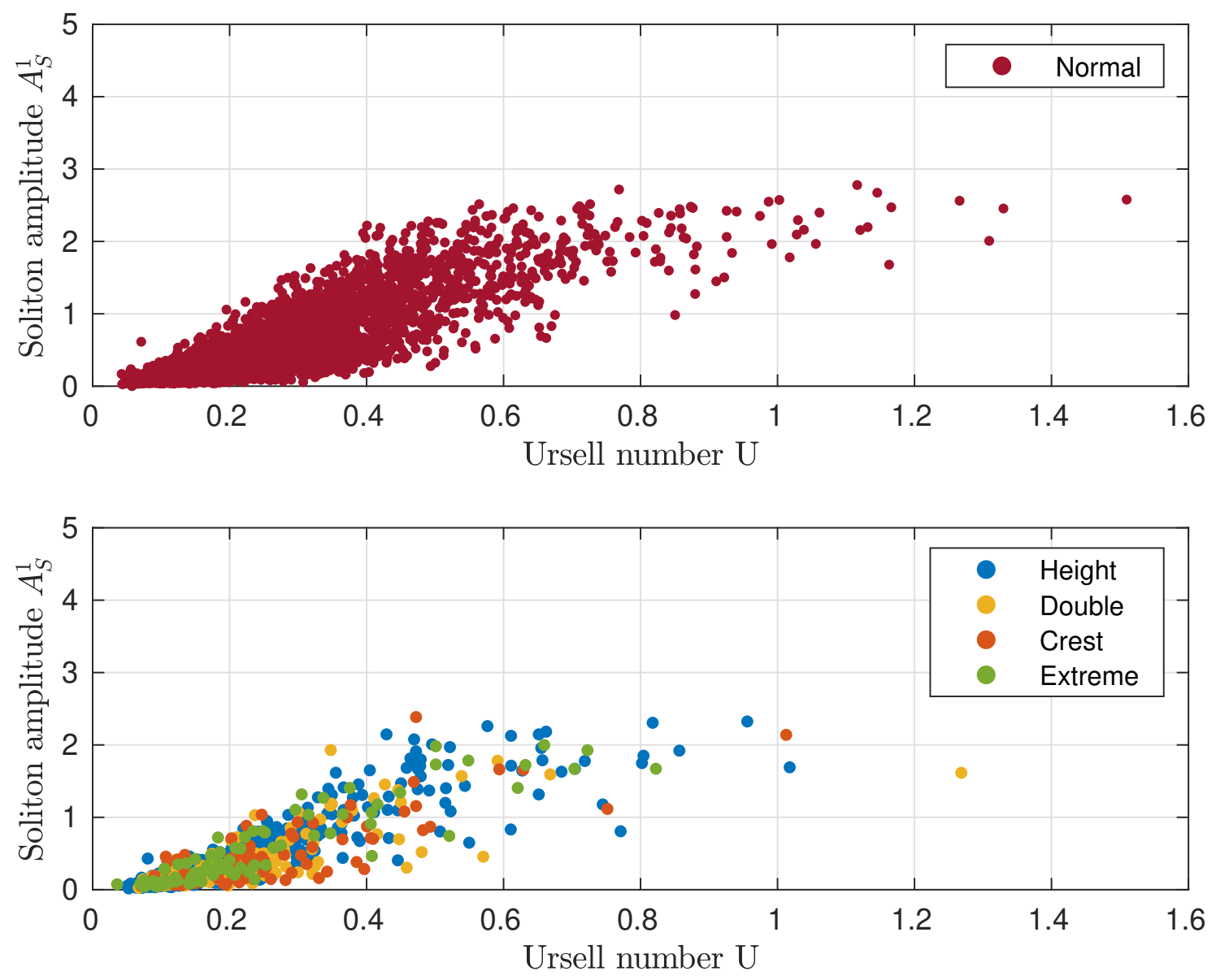

Figure 10. Upper panel: amplitude of the highest soliton attributed to the maximum wave in the time series as a function of the Ursell number of the maximum wave in the time series. Lower panel: amplitude of the highest soliton attributed to the rogue wave as a function of the Ursell number of this rogue wave.

\subsection{Soliton spectra for time series with and without rogue waves}

When investigating the attribution of solitons to rogue waves in Sect. 3.1, we found in the majority of cases that the largest soliton in the nonlinear spectrum could be attributed to the rogue wave. In addition, this soliton was often outstanding from the other solitons in the spectrum, with a much larger amplitude than the remaining solitons in the spectrum (see the example in Fig. 6). We were therefore interested in whether the existence of an outstanding soliton in the nonlinear spectrum was typical for rogue wave samples off Norderney. We investigated this question statistically by comparing soliton spectra, calculated from vKdV-NLFT, for normal samples and the four different categories of rogue wave samples. In fact, while all 15.156 considered time series yielded discrete spectra with a large number of solitons, we identified two characteristic classes of soliton spectra. 
https://doi.org/10.5194/nhess-2022-28

Preprint. Discussion started: 4 March 2022

(c) Author(s) 2022. CC BY 4.0 License.

The typical appearance of a soliton spectrum calculated from a time series without rogue waves, was a cluster of solitons (Fig. 11). On the contrary, soliton spectra calculated from time series including a rogue wave in the majority of cases showed one outstanding soliton with an amplitude much larger than that of the remaining cluster of solitons in the spectrum (Fig. 5).
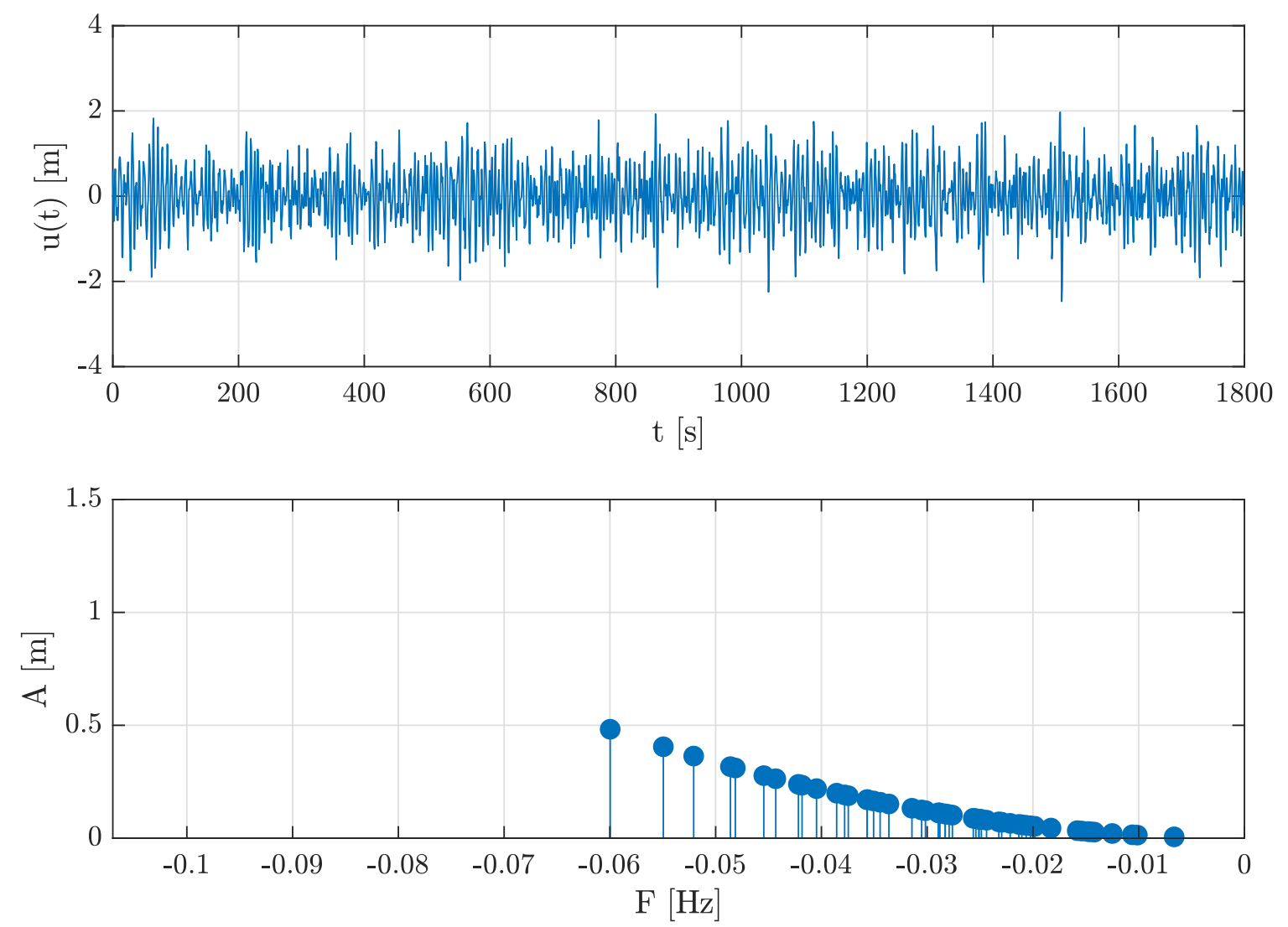

Figure 11. Example of a normal time series without rogue waves, and its corresponding soliton spectrum, calculated from vKdV-NLFT. The soliton spectrum displays a cluster of solitons, found to be typical for the majority of spectra calculated from normal time series. The time series was measured on 26 December 2016, starting at 11:30, with the parameters $H_{\max }=4.44 \mathrm{~m}, H_{s}=2.46 \mathrm{~m}$ and $H_{\max }\left(H_{s}\right)^{-1}=1.80$.

To distinguish between clustered soliton spectra and those featuring an outstanding soliton, we compared the amplitudes of the largest soliton, $A_{1}$, and the second-largest soliton, $A_{2}$, in the discrete spectrum. From the visual inspection of the spectra, we identified a threshold of the ratio $A_{2}\left(A_{1}\right)^{-1}$, below which the largest soliton could be called outstanding:

$\frac{A_{2}}{A_{1}} \leq 0.8$

Thus, a soliton spectrum had an outstanding soliton if the second-largest soliton was at least $20 \%$ smaller than the largest soliton in the spectrum. The choice of this threshold was further supported by the fact that the threshold $A 2(A 1)^{-1}=0.8$ 
https://doi.org/10.5194/nhess-2022-28

Preprint. Discussion started: 4 March 2022

(c) Author(s) 2022. CC BY 4.0 License.

coincides with the median value of $A 2(A 1)^{-1}$ for maximum wave heights just below the rogue wave criterion $H\left(H_{s}\right)^{-1} \geq 2.0$ (Fig. 12). This reveals that our threshold chosen for the distinction between clustered spectra and those featuring an outstanding soliton, at the same time indicates a difference between the spectra calculated from normal and those calculated from rogue wave time series.

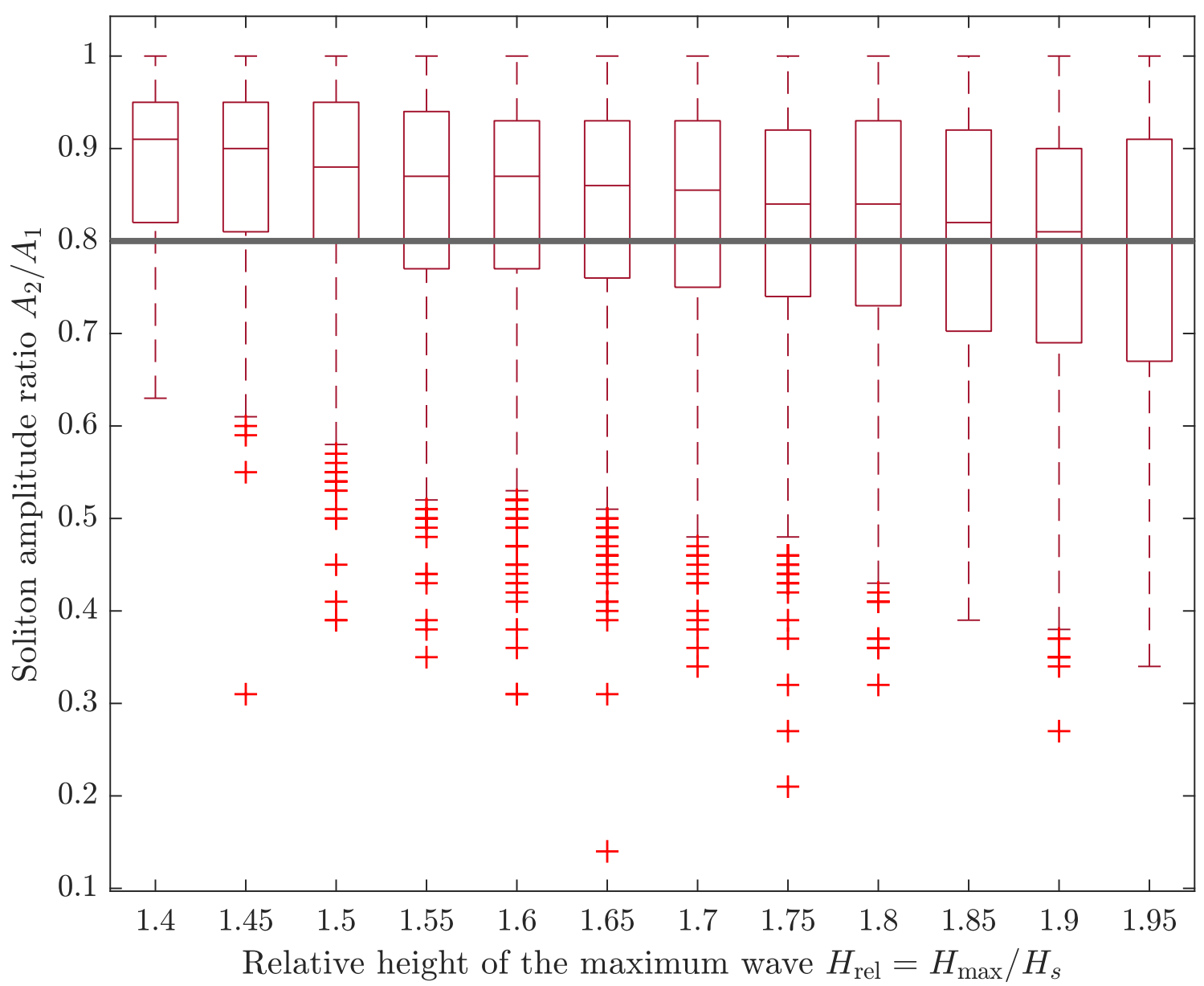

Figure 12. Distribution of the ratio between the second-largest and the largest soliton in the discrete spectrum calculated from normal time series. $H\left(H_{s}\right)^{-1}$ bins of width 0.05 are shown up until $H\left(H_{s}\right)^{-1}<2.0$, which corresponds to the definition of height rogue waves (Eq. (4)). Distributions are shown as box-and-whisker plots (box: interquartile range; whiskers: 1.5 times the interquartile range; horizontal line inside the box: median; red crosses: data outside the whiskers).

Equation (13) is valid for 30 minute samples, which is the standard window size of measurement samples delivered by Datawell Waverider buoys. Since the ratio between soliton amplitudes might be dependent on the window size, it is not clear if 
Eq. (13) would apply to other than 30 minute time windows. The effect of a larger time window size will be discussed in Sect. 4. Table 2 shows the share of outstanding solitons and clustered soliton spectra in each of the categories defined in Sect. 2.1. It is seen that the typical appearance of the soliton spectrum for 30 minute wave measurement samples off Norderney without rogue waves is a cluster of solitons (64\% of the samples), while at the same time it is not unlikely to obtain a soliton spectrum with one outstanding soliton from vKdV-NLFT (36 \% of the samples). For 30 minute rogue wave samples in contrast, it is more likely to obtain a soliton spectrum with one outstanding soliton than a clustered soliton spectrum. This is true for height rogue samples (57\%), and even more pronounced for crest rogue samples (64\%), double rogue samples (72\%) and, finally, extreme rogue samples ( $87 \%$ ). The conclusion can be drawn that the absence of an outstanding soliton is a strong predictor for the absence of an extreme rogue wave. The differences between the four rogue wave categories, indicating that the presence of an outstanding soliton is not equally expressive for all types of rogue waves, may lead to the presumption that not all rogue waves found off Norderney can necessarily be explained by the same theory.

Table 2. Share of samples in each category showing an outstanding soliton or a clustered soliton spectrum, respectively.

\begin{tabular}{cccccc}
\hline & Normal & Height rogue & Crest rogue & Double rogue & Extreme rogue \\
\hline Outstanding soliton & $36 \%$ & $57 \%$ & $64 \%$ & $72 \%$ & $87 \%$ \\
Clustered solitons & $64 \%$ & $43 \%$ & $36 \%$ & $28 \%$ & $13 \%$ \\
\hline
\end{tabular}

The question whether inferences can be made from the time to the spectral domain and vice versa, is answered by a contingency table (Fig. 13). Here, all previously defined rogue wave categories are combined into one joint group of rogue wave samples. Two statements can be made based on the table. On the one hand, the probability that an NLFT spectrum calculated from a normal sample shows an outstanding soliton, is $4986 / 13.984=36 \%$, while the probability that a spectrum calculated from a rogue wave sample shows an outstanding soliton, is $726 / 1172=62 \%$. This indicates that, although not all rogue waves can necessarily be explained by the same theory, outstanding solitons occurred in connection with the majority of observed rogue waves off Norderney. While in the combined group of rogue waves, outstanding solitons play a role in $62 \%$ of the cases, the share differs between the rogue wave categories (Table 2). On the other hand, although rogue waves are more likely to be observed when an outstanding soliton is present in the NLFT spectrum, the presence of an outstanding soliton alone is not sufficient as an indicator for the detection of rogue waves. The main difficulty is the imbalance in sample size between normal samples and rogue wave samples. 


\section{Rogue wave sample (all categories combined)}

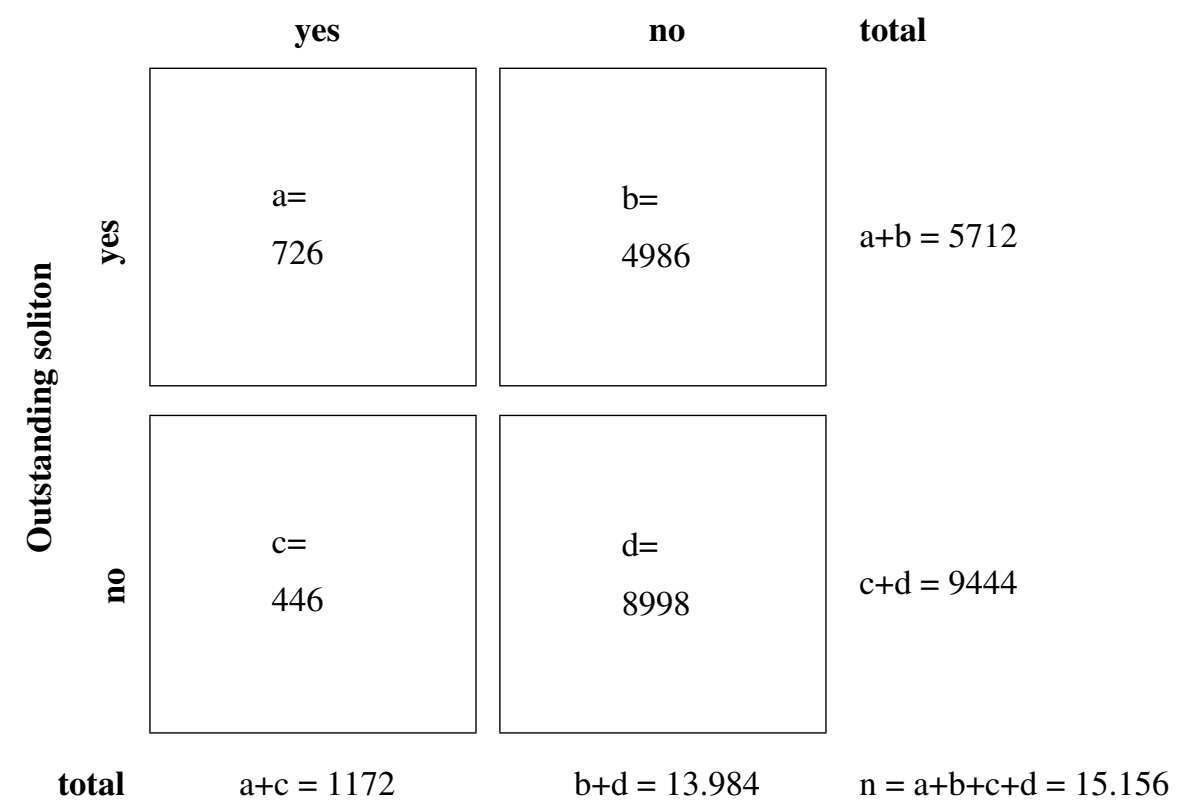

Figure 13. Contingency table of forecast/event pairs. a- hits. b- false alarms. c- misses. d- correct negatives.

In Fig. 14, the ratio between the amplitudes of the second-largest and the largest soliton in the nonlinear spectrum, $A_{2}\left(A_{1}\right)^{-1}$, is visualised in a boxplot for each of the time series categories. A ratio above $A_{2}\left(A_{1}\right)^{-1}=80 \%$, meaning that the secondlargest soliton has a rather similar amplitude to the largest soliton, implies that the soliton spectrum is clustered (Eq. (13)). For normal samples, this is the case for the bulk of time series. The median of the ratio $A_{2}\left(A_{1}\right)^{-1}$ decreases from the most-left to the most-right category on the right axes in Fig. 14. For height rogue waves, the median of $A_{2}\left(A_{1}\right)^{-1}$ is below the $80 \%$-line, with the distribution extending above and below. For double and extreme rogue waves, the gap between the soliton amplitudes may become much larger than for height rogue waves. In some cases, the amplitude $A_{2}$ amounts to less than $30 \%$ of the amplitude $A_{1}$. In all categories except extreme rogue samples, there are samples for which the first and second solitons are almost similar in amplitude $\left(A_{2}\left(A_{1}\right)^{-1} \approx 1\right)$. On the contrary, for all extreme rogue wave samples, $A_{2}$ is below $93 \%$ of $A_{1}$. The large part of soliton spectra from extreme rogue samples shows an outstanding soliton. 


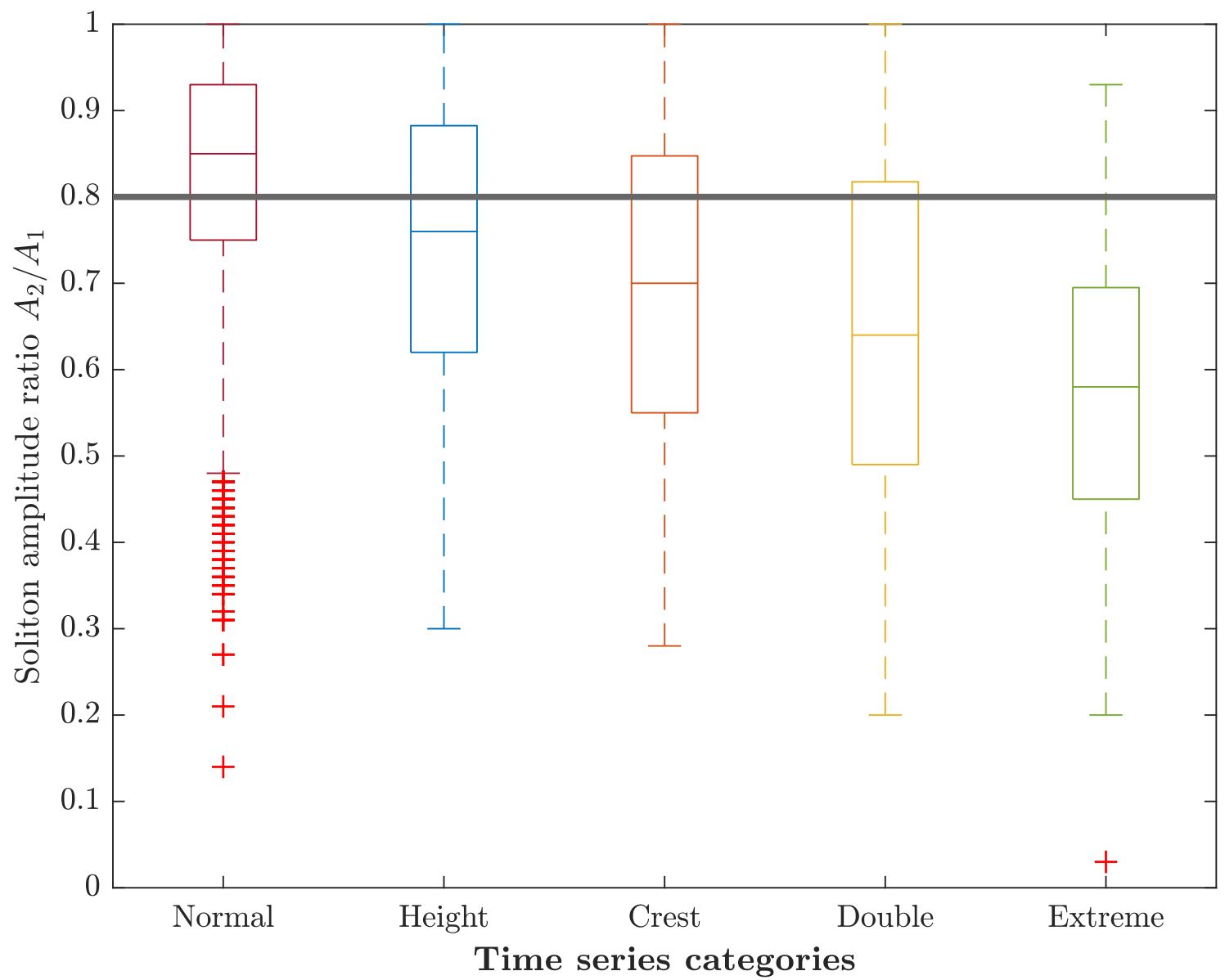

Figure 14. Boxplots of the ratio between the second-largest soliton $\left(A_{2}\right)$ and the largest soliton $\left(A_{1}\right)$ in the spectrum for the different categories of time series. Distributions are shown as box-and-whisker plots (box: interquartile range; whiskers: 1.5 times the interquartile range; horizontal line inside the box: median; red crosses: data outside the whiskers). Below the horizontal line of $80 \%$, the highest soliton in the spectrum is classified as outstanding.

Figure 15 presents the ratio $A_{2}\left(A_{1}\right)^{-1}$ in a scatter plot with one data point for each individual time series. According to this representation, although the presence of an outstanding soliton with $A_{2}\left(A_{1}\right)^{-1} \leq 0.8$ is not a useful indicator of whether a rogue wave is present in the time series or not, the presence of a rogue wave becomes much more likely when one soliton in the nonlinear spectrum is strongly outstanding with $A_{2}\left(A_{1}\right)^{-1} \leq 0.3$ : of all 23 samples satisfying $A_{2}\left(A_{1}\right)^{-1} \leq 0.3$, only $4 / 23=17 \%$ are normal samples, while $19 / 23=83 \%$ of the samples are rogue wave samples ( 1 height, 1 crest, 8 double, 9 extreme rogue wave samples). 
https://doi.org/10.5194/nhess-2022-28

Preprint. Discussion started: 4 March 2022

(c) Author(s) 2022. CC BY 4.0 License.

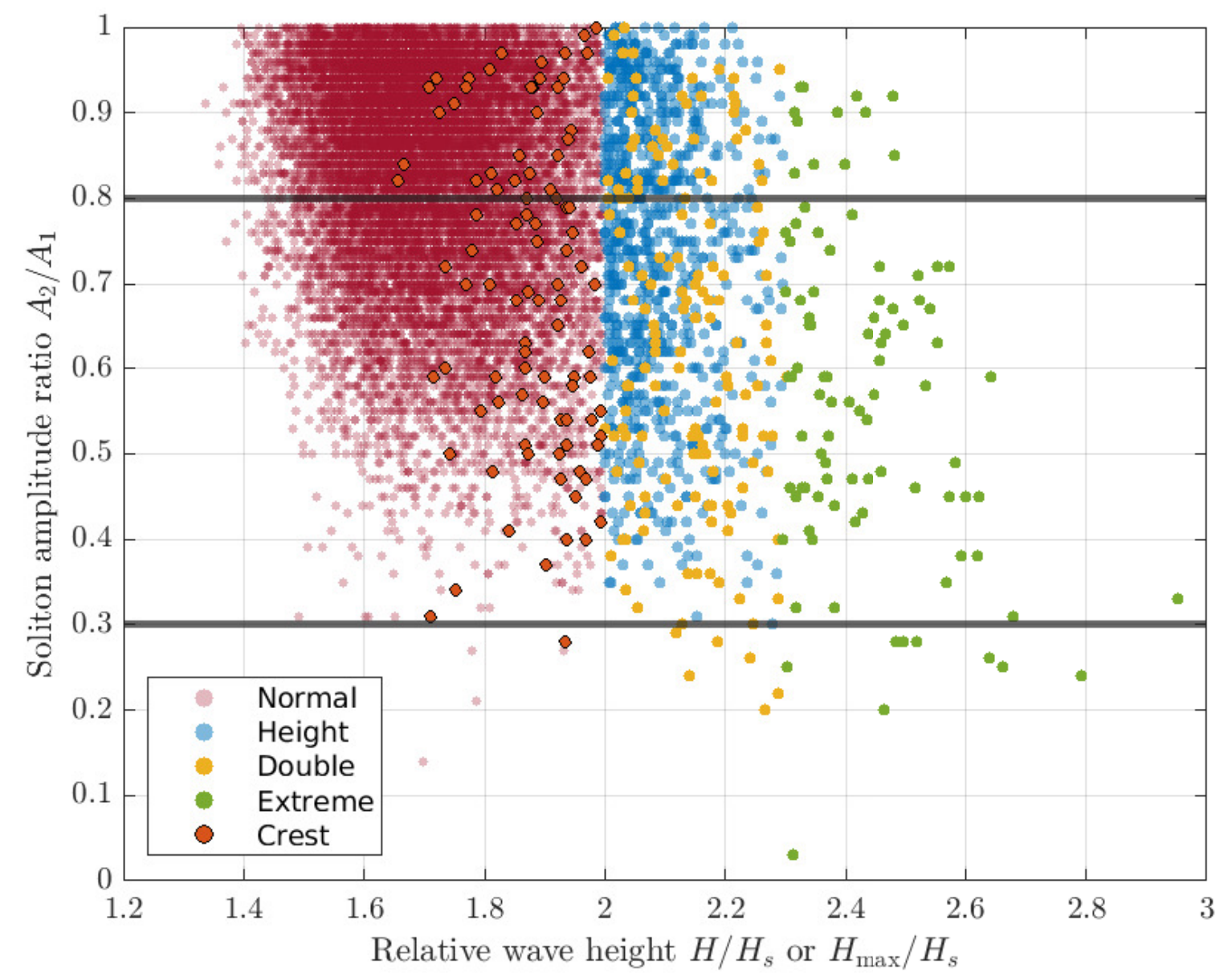

Figure 15. Ratio between the second-largest soliton $\left(A_{2}\right)$ and the largest soliton $\left(A_{1}\right)$ in the spectrum as a function of relative wave height $H\left(H_{s}\right)^{-1}$ or $H_{\max }\left(H_{s}\right)^{-1}$ for the different categories of time series. Below the horizontal line of $80 \%$, the highest soliton in the spectrum is classified as outstanding. Below the horizontal line of $30 \%$, the highest soliton in the spectrum is referred to as strongly outstanding.

\section{Discussion}

We investigated discrete nonlinear soliton spectra obtained by the application of the vKdV-NLFT to time series measured by a surface-following buoy off the coast of the island Norderney in the southern North Sea. The impulse for investigating the data at this specific site by using nonlinear methods was given by a previous study (Teutsch et al., 2020). There, it was found that while second-order distributions were sufficient to describe rogue wave occurrences at nearby stations in somewhat deeper water, the Norderney buoy recorded a larger number of rogue waves than expected according to second-order theory. The results described in this paper suggest that nonlinear processes may explain the enhanced rogue wave occurrence at this 
specific site. The results were derived by the application of vKdV-NLFT and are therefore strictly valid for shallow-water conditions. In a future study, it may be interesting to extend the investigation to additional shallow-water sites.

The bathymetry below the measurement buoy at Norderney is characterized by a strong decrease in water depth. Non-Gaussian wave characteristics as a result of decreasing water depth have already been described e.g. by Huntley et al. (1977)] and gained increased attention in the context of rogue wave occurrence. Increased rogue wave frequencies behind slopes or steps were confirmed by numerous numerical (Sergeeva et al., 2011; Majda et al., 2019; Zhang and Benoit, 2021) and experimental studies (Trulsen et al., 2012; Zeng and Trulsen, 2012; Kashima et al., 2014; Ma et al., 2014; Zhang et al., 2019; Trulsen et al., 2020). Li et al. (2021) have explained the higher occurrence of rogue waves due to an abrupt depth transition from deeper to shallower water by additional second-order free waves generated at the transition. The main subject that the mentioned studies are concerned with, is that waves propagating over a slope, step or bar, are forced into new equilibrium conditions (Zeng and Trulsen, 2012). This mechanism is associated with strong non-Gaussian statistics and an increased rogue wave probability (Zhang and Benoit, 2021). Zeng and Trulsen (2012) anticipate that it may explain the spatially varying occurrence frequency of rogue waves on the continental shelf, where waves enter from the deep sea. Therefore, the described processes associated with a strong decrease in depth might be an explanation for the observed increased rogue wave occurrence off the coast of Norderney (Teutsch et al., 2020). A connection between rogue waves and solitons in this context was established by Sergeeva et al. (2011). The authors showed by applying a KdV equation, that the number of solitons increases in the shallow water behind a slope. They linked this increased soliton occurrence to an increased rogue wave probability. The solutions of the $\mathrm{KdV}$ equation for a given free-surface elevation time series strongly depend on the water depth (see Eq. (7)). While for our calculations, we assumed a constant water depth of $h=10 \mathrm{~m}$, there are in fact major uncertainties regarding the water depth at the actual location of the buoy, due to tidal changes and bathymetry gradients, together with the movement of the buoy, as mentioned in Sect. 2.1 (Fig. 2). The mean tidal range at Norderney is approximately $\pm 1.25 \mathrm{~m}$, while due to an additional movement of the buoy of $2 \mathrm{~m}$ to each side of the slope a total deviation from the nominal water depth of $\pm 2 \mathrm{~m}$ is reasonable. We performed a sensitivity analysis to test the robustness of the results with respect to these uncertainties. To do so, we repeated the computation of the soliton spectrum for water depths of $h=8 \mathrm{~m}$ and $12 \mathrm{~m}$, respectively, while using the same free-surface data as in the previous analysis. A changed water depth leads to different shallow-water conditions (Eq. (3)). For the calculation with a depth of $h=12 \mathrm{~m}$, we repeated the identification of the samples that fulfill shallow-water conditions, as samples and maximum waves due to the larger water depth now had to satisfy the condition $T_{p}$ or $T>5 \mathrm{~s}$, in order to classify as shallowwater samples/ waves. Therefore, only 14.206 samples, that is, approximately $94 \%$ of the original sample size, were available for the calculation at $h=12 \mathrm{~m}$. For the calculation with a depth of $h=8 \mathrm{~m}$, we used the same samples as for the calculation with $h=10 \mathrm{~m}$, because these automatically fulfilled shallow-water conditions at $h=8 \mathrm{~m}$. Irrespective of the water depth adopted in the calculation, the result remained that samples with rogue waves, and especially extreme rogue waves, were more likely to contain an outstanding soliton in the nonlinear spectrum than samples without rogue waves (Table 3). Thus, the results are robust with respect to potential uncertainties in water depth. 
Table 3. Share of samples in each category showing an outstanding soliton in the soliton spectrum, for the respective water depth adopted in the NLFT calculation. Note that for a water depth of $h=12 \mathrm{~m}$, the shallow-water criterion in Eq. (3) changes to $T_{p}>5 \mathrm{~s}$, which left approximately $94 \%$ of the samples for the calculation at a water depth of $12 \mathrm{~m}$.

\begin{tabular}{cccccc}
\hline Water depth & Normal & Height rogue & Crest rogue & Double rogue & Extreme rogue \\
\hline $8 \mathrm{~m}$ & $32 \%$ & $57 \%$ & $61 \%$ & $73 \%$ & $75 \%$ \\
$10 \mathrm{~m}$ & $36 \%$ & $57 \%$ & $64 \%$ & $72 \%$ & $87 \%$ \\
$12 \mathrm{~m}$ & $36 \%$ & $53 \%$ & $62 \%$ & $70 \%$ & $76 \%$ \\
\hline
\end{tabular}

The KdV equation is only valid for unidirectional waves. Although Osborne (1993) recommends the application of the NLFT for $\mathrm{KdV}$ to measurement data only for samples in which the largest part of the energy is in the dominant propagation direction, we applied the KdV-NLFT outside the limits that are given in the literature. At our measurement site, the sea state was always multidirectional, with a directional spreading of the wave energy approximately between $28^{\circ}$ and $55^{\circ}$, while in the dataset of Osborne (1993), only $5 \%$ of the energy were perpendicular to the dominant direction of propagation. We repeated the first part of the analysis, for which the results are described in Sect. 3.1, for the approximately $10 \%$ of samples in each category with the lowest directional spread. This corresponded to a threshold in directional spread of $35^{\circ}$ for most categories, except crest rogue waves, which tended to occur in broader sea states (threshold at $36.5^{\circ}$ ) and extreme rogue waves, which statistically occur in more narrow sea states (Christou and Ewans, 2014) (threshold at $34^{\circ}$ ). We found our result- that an outstanding soliton is more typical for a rogue wave time series than for a normal time series- confirmed and partly emphasised (Table 4). Therefore, we rate vKdV-NLFT, although assuming unidirectionality in multidirectional measurement samples, an appropriate tool to evaluate the connection between solitons and rogue waves off Norderney.

Table 4. Share of samples in each category showing an outstanding soliton, for the approximately $10 \%$ most narrow samples.

\begin{tabular}{cccccc}
\hline & Normal & Height rogue & Crest rogue & Double rogue & Extreme rogue \\
\hline No. of samples & 1614 & 91 & 12 & 17 & 10 \\
Outstanding soliton & $31 \%$ & $57 \%$ & $67 \%$ & $88 \%$ & $90 \%$ \\
\hline
\end{tabular}

We would like to put an emphasis on the limitation of our suggested definition of an outstanding soliton (Eq. (13)) to the size of the measurement window. Our criterion was chosen based on the inspection of soliton spectra from 30 minute time series. However, the gap size might change depending on the chosen window size. An increase in window size, meaning more waves in the time series, will introduce additional solitons to the spectrum. If these are larger than $A_{1}$ or emerge in between $A_{1}$ and $A_{2}$, the gap size between the two largest solitons will be influenced. If these are smaller than $A_{2}$, their emergence will not alter the gap between $A_{1}$ and $A_{2}$. Similarly, a reduction in window size would exclude waves in the time series and remove solitons corresponding to these waves. If this modification leads to the removal of the largest or second-largest soliton, the gap between the new $A_{1}$ and $A_{2}$ will become larger or smaller than for a 30 minute time window. If this modification only affects solitons smaller than $A_{2}$, the size of the gap between $A_{1}$ and $A_{2}$ will not be influenced. We applied the ratio between $A_{2}$ and $A_{1}$ merely 
as a measure to statistically evaluate differences in the soliton spectra calculated from 30 minute normal and rogue wave time series. For different window sizes, it might be necessary to define new criteria.

Our result that rogue wave samples have a higher probability of showing an outstanding soliton in the nonlinear spectrum compared to normal samples becomes most obvious in the categories of double and extreme rogue samples. In these categories, differences from normal samples are visible not only in the percentage of outstanding solitons, but also in the magnitude of the amplitude gap between the first and second solitons in the spectrum. Height rogue waves, on the contrary, do not seem to differ very much from high waves in normal samples, both in terms of the gap between first and second soliton in the spectrum, and the height of the solitons associated with the maximum wave. The fact that differences between time series with and without rogue waves become apparent only in some of the chosen categories, raises the question whether the choice of rogue wave definitions has been reasonable for the considered location. The rogue wave definitions serving as a basis to this study have been introduced by Haver and Andersen (2000) for deep water waves. The relative height and crest values in their definitions represent outliers, being exceeded in 1 of 100 cases when applying a second-order model to the deep-water sea-surface elevation (Haver, 2000). The definitions have been taken up numerous times in the literature. Authors have been investigating whether rogue waves according to Haver and Andersen's definition (2000) are outliers with respect to typical wave distributions in the real ocean as well (e.g., Forristall, 2005; Gemmrich and Garrett, 2008). The question has been raised whether the rogue wave definition by a certain height or crest threshold is useful in practice (Häfner et al., 2021). Several authors have, based on large measurement datasets, come to the conclusion that these rogue waves are rare, but nevertheless realisations of commonly used wave distributions (e.g., Waseda et al., 2011; Christou and Ewans, 2014). In a previous study (Teutsch et al., 2020), we were able to confirm this conclusion at buoy measurement stations in intermediate water. However, at the shallow water buoy station off Norderney, which showed a larger number of rogue waves than expected according to the common wave distributions, the interaction of solitons with oscillating waves might be a mechanism explaining the increased occurrence of rogue waves.

\section{Conclusions}

Rogue wave occurrence recorded off the coast of the island Norderney is not sufficiently explained by second-order theory. We investigated the role of solitons in the enhanced rogue wave occurrence by calculating discrete soliton spectra of time series from vKdV-NLFT. Our main results for this specific measurement site are the following.

- Each measured rogue wave could be associated with at least one soliton in the NLFT spectrum.

- The soliton heights were always smaller than those of the rogue waves. Samples with rogue waves were more likely to contain an outstanding soliton in the NLFT spectrum than samples without rogue waves.

- The presence of a strongly outstanding soliton, with a ratio between the second-largest and the largest soliton in the nonlinear spectrum of $A_{2}\left(A_{1}\right)^{-1} \leq 0.3$, was found to be a strong indicator for the presence of a rogue wave. 
https://doi.org/10.5194/nhess-2022-28

Preprint. Discussion started: 4 March 2022

(C) Author(s) 2022. CC BY 4.0 License.

(c) (i)

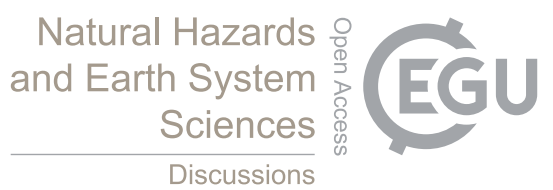

- Conversely, the absence of an outstanding soliton in the spectrum is a strong indicator for the absence of an extreme rogue wave of $H\left(H_{s}\right)^{-1} \geq 2.3$.

We conclude that nonlinear processes are important in the generation of rogue waves at this specific site and may explain the enhanced occurrence of such waves beyond second-order theory. Rogue waves at Norderney are likely to be a result of the interaction of solitons with the underlying field of oscillatory waves.

Author contributions. All authors contributed to the idea and scope of the paper. IT performed the analyses and wrote the manuscript. MB, RW and SW provided help with data analysis, discussed the results, and contributed to the writing of the paper. RW supervised the work.

Competing interests. The authors declare that they have no conflict of interest.

465 Acknowledgements. This project has received funding from the European Research Council (ERC) under the European Union's Horizon 2020 research and innovation programme (grant agreement No 716669). Ina Teutsch received funding for this work from the Federal Maritime and Hydrographic Agency (BSH). The buoy data were kindly provided by the Lower Saxony Water Management, Coastal Defence and Nature Conservation Agency (NLWKN). 
https://doi.org/10.5194/nhess-2022-28

Preprint. Discussion started: 4 March 2022

(c) Author(s) 2022. CC BY 4.0 License.

\section{References}

Ablowitz, M. J., Kaup, D. J., Newell, A. C., and Segur, H.: The Inverse Scattering Transform-Fourier Analysis for Nonlinear Problems, Studies in Applied Mathematics, 53, 249-315, https://doi.org/10.1002/sapm1974534249, 1974.

Alber, I. E.: The effects of randomness on the stability of two-dimensional surface wavetrains, Proceedings of the Royal Society of London. A. Mathematical and Physical Sciences, 363, 525-546, https://doi.org/10.1098/rspa.1978.0181, 1978.

Baschek, B. and Imai, J.: Rogue Wave Observations Off the US West Coast, Oceanography, 24, 158-165, https://doi.org/10.5670/oceanog.2011.35, 2011.

Benjamin, T. B. and Feir, J. E.: The disintegration of wave trains on deep water, Journal of Fluid Mechanics, 27, 417-430, https://doi.org/10.1017/s002211206700045x, 1967.

Bitner, E. M.: Non-linear effects of the statistical model of shallow-water wind waves, Applied Ocean Research, 2, 63-73, https://doi.org/10.1016/0141-1187(80)90031-0, 1980.

Brühl, M. and Oumeraci, H.: Analysis of long-period cosine-wave dispersion in very shallow water using nonlinear Fourier transform based on KdV equation, Applied Ocean Research, 61, 81-91, https://doi.org/10.1016/j.apor.2016.09.009, 2016.

Brühl, M.: Spectral analysis of nonlinear waves in the coastal area, 2022.

Cattrell, A. D., Srokosz, M., Moat, B. I., and Marsh, R.: Can Rogue Waves Be Predicted Using Characteristic Wave Parameters?, Journal of Geophysical Research: Oceans, 123, 5624-5636, https://doi.org/10.1029/2018jc013958, 2018.

Christou, M. and Ewans, K.: Field Measurements of Rogue Water Waves, Journal of Physical Oceanography, 44, 2317-2335, https://doi.org/10.1175/jpo-d-13-0199.1, 2014.

Clamond, D., Francius, M., Grue, J., and Kharif, C.: Long time interaction of envelope solitons and freak wave formations, European Journal of Mechanics - B/Fluids, 25, 536-553, https://doi.org/10.1016/j.euromechflu.2006.02.007, 2006.

de León, S. P. and Soares, C. G.: Hindcast of extreme sea states in North Atlantic extratropical storms, Ocean Dynamics, 65, 241-254, https://doi.org/10.1007/s10236-014-0794-6, 2014.

Dysthe, K., Krogstad, H. E., and Müller, P.: Oceanic Rogue Waves, Annual Review of Fluid Mechanics, 40, 287-310, https://doi.org/10.1146/annurev.fluid.40.111406.102203, 2008.

Dysthe, K. B. and Trulsen, K.: Note on Breather Type Solutions of the NLS as Models for Freak-Waves, Physica Scripta, T82, 48, https://doi.org/10.1238/physica.topical.082a00048, 1999.

Fedele, F., Herterich, J., Tayfun, A., and Dias, F.: Large nearshore storm waves off the Irish coast, Scientific Reports, 9, https://doi.org/10.1038/s41598-019-51706-8, 2019.

Fernandez, L., Onorato, M., Monbaliu, J., and Toffoli, A.: Modulational instability and wave amplification in finite water depth, Natural Hazards and Earth System Sciences, 14, 705-711, https://doi.org/10.5194/nhess-14-705-2014, 2014.

Forristall, G. Z.: On the statistical distribution of wave heights in a storm, Journal of Geophysical Research, 83, 2353, https://doi.org/10.1029/jc083ic05p02353, 1978.

Forristall, G. Z.: Understanding rogue waves: Are new physics really necessary?, 2005.

Gardner, C. S., Greene, J. M., Kruskal, M. D., and Miura, R. M.: Method for solving the Korteweg-deVries equation., Phys. Rev. Lett., 19: 1095-7(Nov. 6, 1967)., https://doi.org/10.1103/PhysRevLett.19.1095, 1967.

Garett, C. and Gemmrich, J.: Rogue waves, Physics Today, 62, 62-63, https://doi.org/10.1063/1.3156339, 2009. 
https://doi.org/10.5194/nhess-2022-28

Preprint. Discussion started: 4 March 2022

(c) Author(s) 2022. CC BY 4.0 License.

(c) (i)

Gemmrich, J. and Garrett, C.: Unexpected Waves, Journal of Physical Oceanography, 38, 2330-2336, https://doi.org/10.1175/2008jpo3960.1, 2008.

Gramstad, O. and Trulsen, K.: Influence of crest and group length on the occurrence of freak waves, Journal of Fluid Mechanics, 582, 463-472, https://doi.org/10.1017/s0022112007006507, 2007.

Häfner, D., Gemmrich, J., and Jochum, M.: Real-world rogue wave probabilities, Scientific Reports, 11, https://doi.org/10.1038/s41598-02189359-1, 2021.

Hammack, J., Scheffner, N., and Segur, H.: Two-dimensional periodic waves in shallow water, Journal of Fluid Mechanics, 209, 567-589, https://doi.org/10.1017/s0022112089003228, 1989.

Haver, S.: Evidences of the existence of freak waves, in: Proc. Rogue Waves, Brest, 2000.

Haver, S. and Andersen, O. J.: Freak waves: rare realizations of a typical population or typical realizations of a rare population?, in: The Tenth International Offshore and Polar Engineering Conference, International Society of Offshore and Polar Engineers, 2000.

Huntley, D. A., Guza, R. T., and Bowen, A. J.: A universal form for shoreline run-up spectra?, Journal of Geophysical Research, 82, 25772581, https://doi.org/10.1029/jc082i018p02577, 1977.

Janssen, P. A. E. M.: Nonlinear Four-Wave Interactions and Freak Waves, Journal of Physical Oceanography, 33, 863-884, 2003.

Janssen, P. A. E. M. and Onorato, M.: The Intermediate Water Depth Limit of the Zakharov Equation and Consequences for Wave Prediction, Journal of Physical Oceanography, 37, 2389-2400, https://doi.org/10.1175/jpo3128.1, 2007.

Johnson, D.: DIWASP, a directional wave spectra toolbox for MATLAB®: User Manual, Centre for Water Research, University of Western Australia., 2002.

Karmpadakis, I., Swan, C., and Christou, M.: Laboratory investigation of crest height statistics in intermediate water depths, Proceedings of the Royal Society A: Mathematical, Physical and Engineering Sciences, 475, 20190 183, https://doi.org/10.1098/rspa.2019.0183, 2019.

Karmpadakis, I., Swan, C., and Christou, M.: Assessment of wave height distributions using an extensive field database, Coastal Engineering, 157, 103 630, https://doi.org/10.1016/j.coastaleng.2019.103630, 2020.

Kashima, H., Hirayama, K., and Mori, N.: ESTIMATION OF FREAK WAVE OCCURRENCE FROM DEEP TO SHALLOW WATER REGIONS, Coastal Engineering Proceedings, 1, 36, https://doi.org/10.9753/icce.v34.waves.36, 2014.

Kharif, C. and Pelinovsky, E.: Physical mechanisms of the rogue wave phenomenon, European Journal of Mechanics - B/Fluids, 22, 603-634, https://doi.org/10.1016/j.euromechflu.2003.09.002, 2003.

Korteweg, D. J. and de Vries, G.: XLI. On the change of form of long waves advancing in a rectangular canal, and on a new type of long stationary waves, The London, Edinburgh, and Dublin Philosophical Magazine and Journal of Science, 39, 422-443, https://doi.org/10.1080/14786449508620739, 1895.

LeMéhauté, B.: An introduction to hydrodynamics and water waves, Springer-Verlag, New York, 1976.

535 Lenau, C. W.: The solitary wave of maximum amplitude, Journal of Fluid Mechanics, 26, 309-320, https://doi.org/10.1017/s0022112066001253, 1966.

Li, Y., Draycott, S., Zheng, Y., Lin, Z., Adcock, T. A., and van den Bremer, T. S.: Why rogue waves occur atop abrupt depth transitions, Journal of Fluid Mechanics, 919, R5, https://doi.org/10.1017/jfm.2021.409, 2021.

Longuet-Higgins, M. S.: On the Statistical Distribution of the Height of Sea Waves, Journal of Marine Research, $11,1952$.

540 Ma, Y., Dong, G., and Ma, X.: EXPERIMENTAL STUDY OF STATISTICS OF RANDOM WAVES PROPAGATING OVER A BAR, Coastal Engineering Proceedings, 1, 30, https://doi.org/10.9753/icce.v34.waves.30, 2014. 
https://doi.org/10.5194/nhess-2022-28

Preprint. Discussion started: 4 March 2022

(c) Author(s) 2022. CC BY 4.0 License.

Majda, A. J., Moore, M. N. J., and Qi, D.: Statistical dynamical model to predict extreme events and anomalous features in shallow water waves with abrupt depth change, Proceedings of the National Academy of Sciences, 116, 3982-3987, https://doi.org/10.1073/pnas.1820467116, 2019.

545 MATLAB: version 9.6.0.1072779 (R2019a), The MathWorks Inc., Natick, Massachusetts, 2019.

McCowan, J.: VII. On the solitary wave, The London, Edinburgh, and Dublin Philosophical Magazine and Journal of Science, 32, 45-58, https://doi.org/10.1080/14786449108621390, 1891.

Middleton, D. and Mellen, R.: Wind-generated solitons: A potentially significant mechanism in ocean surface wave generation and surface scattering, IEEE Journal of Oceanic Engineering, 10, 471-476, https://doi.org/10.1109/JOE.1985.1145130, 1985.

550 Miles, J. W.: Solitary Waves, Annual Review of Fluid Mechanics, 12, 11-43, https://doi.org/10.1146/annurev.fl.12.010180.000303, 1980.

NLWKN: Tideaußenpegel, https://www.pegelonline.nlwkn.niedersachsen.de/Pegel/TideaulT1\ssenpegel/ID/452, accessed: 2021-12-23, 2021.

Olagnon, M. and v. Iseghem, S.: Some observed characteristics of sea states with extreme waves, in: Proc. 10th Int. Offshore Polar Engineering Conf., Seattle, pp. 84-90, International Society of Offshore and Polar Engineers, 2000.

Onorato, M., Osborne, A. R., Serio, M., and Bertone, S.: Freak Waves in Random Oceanic Sea States, Physical Review Letters, 86, 58315834, https://doi.org/10.1103/physrevlett.86.5831, 2001.

Orzech, M. D. and Wang, D.: Measured Rogue Waves and Their Environment, Journal of Marine Science and Engineering, 8, 890, https://doi.org/10.3390/jmse8110890, 2020.

Osborne, A. R.: Behavior of solitons in random-function solutions of the periodic Korteweg-de Vries equation, Physical Review Letters, 71, 3115-3118, https://doi.org/10.1103/physrevlett.71.3115, 1993.

Osborne, A. R.: Nonlinear ocean waves and the inverse scattering transform, Elsevier, Amsterdam, 2010.

Osborne, A. R., Segre, E., Boffetta, G., and Cavaleri, L.: Soliton basis states in shallow-water ocean surface waves, Physical Review Letters, 67, 592-595, https://doi.org/10.1103/physrevlett.67.592, 1991.

Pelinovsky, E. and Sergeeva, A.: Numerical modeling of the KdV random wave field, European Journal of Mechanics - B/Fluids, 25, 425434, https://doi.org/10.1016/j.euromechflu.2005.11.001, 2006.

Pelinovsky, E., Talipova, T., and Kharif, C.: Nonlinear-dispersive mechanism of the freak wave formation in shallow water, Physica D: Nonlinear Phenomena, 147, 83-94, https://doi.org/10.1016/s0167-2789(00)00149-4, 2000.

Peregrine, D. H.: Water waves, nonlinear Schrödinger equations and their solutions, The Journal of the Australian Mathematical Society. Series B. Applied Mathematics, 25, 16-43, https://doi.org/10.1017/S0334270000003891, 1983.

570 Peterson, P., Soomere, T., Engelbrecht, J., and van Groesen, E.: Soliton interaction as a possible model for extreme waves in shallow water, Nonlinear Processes in Geophysics, 10, 503-510, https://doi.org/10.5194/npg-10-503-2003, 2003.

Pinho, U., Liu, P., Eduardo, C., and Ribeiro, C.: Freak Waves at Campos Basin, Brazil, Geofizika, 21, 2004.

Prevosto, M.: Effect of Directional Spreading and Spectral Bandwidth on the Nonlinearity of the Irregular Waves, 1998.

Sergeeva, A., Pelinovsky, E., and Talipova, T.: Nonlinear random wave field in shallow water: variable Korteweg-de Vries framework, Natural

Hazards and Earth System Sciences, 11, 323-330, https://doi.org/10.5194/nhess-11-323-2011, 2011.

Serio, M., Onorato, M., Osborne, A., and Janssen, P.: On the computation of the Benjamin-Feir Index, Il Nuovo Cimento C, 28, 893-903, https://doi.org/10.1393/ncc/i2005-10134-1, 2006.

Shrira, V. I. and Geogjaev, V. V.: What makes the Peregrine soliton so special as a prototype of freak waves?, Journal of Engineering Mathematics, 67, 11-22, https://doi.org/10.1007/s10665-009-9347-2, 2009. 
https://doi.org/10.5194/nhess-2022-28

Preprint. Discussion started: 4 March 2022

(c) Author(s) 2022. CC BY 4.0 License.

(c) (i)

Slunyaev, A., Didenkulova, I., and Pelinovsky, E.: Rogue waters, Contemporary Physics, 52, 571-590, https://doi.org/10.1080/00107514.2011.613256, 2011.

Slunyaev, A., Sergeeva, A., and Didenkulova, I.: Rogue events in spatiotemporal numerical simulations of unidirectional waves in basins of different depth, Natural Hazards, 84, 549-565, https://doi.org/10.1007/s11069-016-2430-x, 2016.

Slunyaev, A. V.: A high-order nonlinear envelope equation for gravity waves in finite-depth water, Journal of Experimental and Theoretical Physics, 101, 926-941, https://doi.org/10.1134/1.2149072, 2005.

Slunyaev, A. V. and Shrira, V. I.: On the highest non-breaking wave in a group: fully nonlinear water wave breathers versus weakly nonlinear theory, Journal of Fluid Mechanics, 735, 203-248, https://doi.org/10.1017/jfm.2013.498, 2013.

Soares, C. G., Cherneva, Z., and Antão, E.: Characteristics of abnormal waves in North Sea storm sea states, Applied Ocean Research, 25, 337-344, https://doi.org/10.1016/j.apor.2004.02.005, 2003.

590 Soomere, T.: Rogue waves in shallow water, The European Physical Journal Special Topics, 185, 81-96, https://doi.org/10.1140/epjst/e201001240-1, 2010.

Soomere, T. and Engelbrecht, J.: Interaction of shallow-water solitons as a possible model for freak waves, 2005.

Stansell, P.: Distributions of freak wave heights measured in the North Sea, Applied Ocean Research, 26, 35-48, https://doi.org/10.1016/j.apor.2004.01.004, 2004.

Tayfun, M. A.: Distributions of Envelope and Phase in Wind Waves, Journal of Physical Oceanography, 38, 2784-2800, https://doi.org/10.1175/2008jpo4008.1, 2008.

Teutsch, I., Weisse, R., Moeller, J., and Krueger, O.: A statistical analysis of rogue waves in the southern North Sea, Natural Hazards and Earth System Sciences, 20, 2665-2680, https://doi.org/10.5194/nhess-20-2665-2020, 2020.

Toffoli, A., Fernandez, L., Monbaliu, J., Benoit, M., Gagnaire-Renou, E., Lefèvre, J. M., Cavaleri, L., Proment, D., Pakozdi, C., Stansberg,

C. T., Waseda, T., and Onorato, M.: Experimental evidence of the modulation of a plane wave to oblique perturbations and generation of rogue waves in finite water depth, Physics of Fluids, 25, 091 701, https://doi.org/10.1063/1.4821810, 2013.

Trulsen, K., Zeng, H., and Gramstad, O.: Laboratory evidence of freak waves provoked by non-uniform bathymetry, Physics of Fluids, 24, 097 101, https://doi.org/10.1063/1.4748346, 2012.

Trulsen, K., Raust $\varnothing 1$, A., Jorde, S., and Rye, L. B.: Extreme wave statistics of long-crested irregular waves over a shoal, Journal of Fluid Mechanics, 882, R2, https://doi.org/10.1017/jfm.2019.861, 2020.

Ursell, F.: The long-wave paradox in the theory of gravity waves, Mathematical Proceedings of the Cambridge Philosophical Society, 49, 685-694, https://doi.org/10.1017/s0305004100028887, 1953.

Wahls, S. and Poor, H. V.: Fast Numerical Nonlinear Fourier Transforms, IEEE Transactions on Information Theory, 61, 6957-6974, https://doi.org/10.1109/tit.2015.2485944, 2015.

610 Wahls, S., Chimmalgi, S., and Prins, P. J.: FNFT: A Software Library for Computing Nonlinear Fourier Transforms, Journal of Open Source Software, 3, 597, https://doi.org/10.21105/joss.00597, 2018.

Waseda, T., Hallerstig, M., Ozaki, K., and Tomita, H.: Enhanced freak wave occurrence with narrow directional spectrum in the North Sea, Geophysical Research Letters, 38, n/a-n/a, https://doi.org/10.1029/2011g1047779, 2011.

Zabusky, N. J. and Kruskal, M. D.: Interaction of "Solitons" in a Collisionless Plasma and the Recurrence of Initial States, Physical Review Letters, 15, 240-243, https://doi.org/10.1103/physrevlett.15.240, 1965.

Zakharov, V. E. and Shabat, A. B.: A scheme for integrating the nonlinear equations of mathematical physics by the method of the inverse scattering problem. I, Functional Analysis and Its Applications, 8, 226-235, https://doi.org/10.1007/bf01075696, 1975. 
https://doi.org/10.5194/nhess-2022-28

Preprint. Discussion started: 4 March 2022

(c) Author(s) 2022. CC BY 4.0 License.

(c) (1)

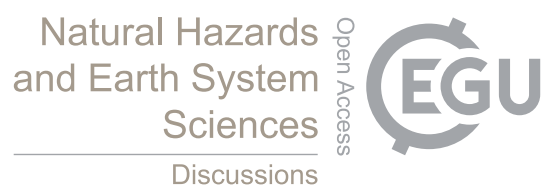

Zeng, H. and Trulsen, K.: Evolution of skewness and kurtosis of weakly nonlinear unidirectional waves over a sloping bottom, Natural Hazards and Earth System Sciences, 12, 631-638, https://doi.org/10.5194/nhess-12-631-2012, 2012.

Zhang, J. and Benoit, M.: Wave-bottom interaction and extreme wave statistics due to shoaling and de-shoaling of irregular long-crested wave trains over steep seabed changes, Journal of Fluid Mechanics, 912, https://doi.org/10.1017/jfm.2020.1125, 2021.

Zhang, J., Benoit, M., Kimmoun, O., Chabchoub, A., and Hsu, H.-C.: Statistics of Extreme Waves in Coastal Waters: Large Scale Experiments and Advanced Numerical Simulations, Fluids, 4, 99, https://doi.org/10.3390/fluids4020099, 2019. 